ARTICLE

\title{
Discovery of fungal oligosaccharide-oxidising flavo-enzymes with previously unknown substrates, redox-activity profiles and interplay with LPMOs
}

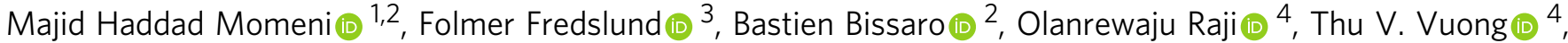 \\ Sebastian Meier (1) ${ }^{5}$, Tine Sofie Nielsen', Vincent Lombard ${ }^{6}$, Bruno Guigliarelli ${ }^{7}$, Frédéric Biaso ${ }^{7}$, Mireille Haon $^{2}$, \\ Sacha Grisel ${ }^{2}$, Bernard Henrissat (10,8,9, Ditte Hededam Welner (1D ${ }^{3}$, Emma R. Master ${ }^{4,10}$, Jean-Guy Berrin ${ }^{2 \otimes} \&$ \\ Maher Abou Hachem (1D ${ }^{1 凶}$
}

Oxidative plant cell-wall processing enzymes are of great importance in biology and biotechnology. Yet, our insight into the functional interplay amongst such oxidative enzymes remains limited. Here, a phylogenetic analysis of the auxiliary activity 7 family (AA7), currently harbouring oligosaccharide flavo-oxidases, reveals a striking abundance of AA7-genes in phytopathogenic fungi and Oomycetes. Expression of five fungal enzymes, including three from unexplored clades, expands the AA7-substrate range and unveils a cellooligosaccharide dehydrogenase activity, previously unknown within AA7. Sequence and structural analyses identify unique signatures distinguishing the strict dehydrogenase clade from canonical AA7 oxidases. The discovered dehydrogenase directly is able to transfer electrons to an AA9 lytic polysaccharide monooxygenase (LPMO) and fuel cellulose degradation by LPMOs without exogenous reductants. The expansion of redox-profiles and substrate range highlights the functional diversity within AA7 and sets the stage for harnessing AA7 dehydrogenases to fine-tune LPMO activity in biotechnological conversion of plant feedstocks.

\footnotetext{
${ }^{1}$ Department of Biotechnology and Biomedicine, Technical University of Denmark, Lyngby, Denmark. ${ }^{2}$ INRAE, Aix Marseille Univ, Biodiversité et Biotechnologie Fongiques (BBF), Marseille, France. ${ }^{3}$ The Novo Nordisk Center for Biosustainability, Lyngby, Denmark. ${ }^{4}$ Department of Chemical Engineering and Applied Chemistry, University of Toronto, Toronto, ON, Canada. ${ }^{5}$ Department of Chemistry, Technical University of Denmark, Lyngby, Denmark. ${ }^{6}$ Architecture et Fonction des Macromolécules Biologiques, UMR 7257 CNRS, USC 1408, Aix Marseille Univ, Marseille, France. ${ }^{7}$ Aix-Marseille Univ, CNRS, UMR7281 Unité de Bioénergétique et Ingénierie des Protéines (BIP), Marseille, France. ${ }^{8}$ INRAE, USC1408 Architecture et Fonction des Macromolécules Biologiques (AFMB), Marseille, France. ${ }^{9}$ Department of Biological Sciences, King Abdulaziz University, Jeddah, Saudi Arabia. ${ }^{10}$ Department of Bioproducts

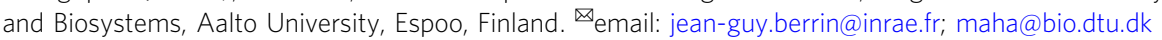


T he involvement of oxidative processes in polysaccharide degradation by fungi has been proposed by the pioneering work of Eriksson et al. in 1974 ${ }^{1}$. This notion has gained strong support by the recent discovery of lytic polysaccharide monooxygenases (LPMOs) that uniquely catalyse the oxidative cleavage of glycosidic bonds in (semi)crystalline polysaccharides such as starch ${ }^{2-4}$, chitin ${ }^{5}$, cellulose ${ }^{6-8}$ and cellulose-bound hemicelluloses, e.g., xyloglucan ${ }^{9}$ and xylan ${ }^{10}$. Besides LPMOs, filamentous fungi co-secrete an impressing diversity of carbohydrate-specific oxidoreductases ${ }^{11}$. To date, only four fungal oligosaccharide-oxidising enzymes from the auxiliary activity family 7 (AA7) in the Carbohydrate Active enZyme (CAZy) database $^{12}$, have been characterized. In addition, oligosaccharide oxidases from plants have been reported ${ }^{13,14}$, but are currently not assigned into AA7. Our insight is, thus, clearly limited regarding the biological roles as well as the diversity of substrates and redox features within this family. Fungal AA7 enzymes catalyse the oxidation of the reducing end $\mathrm{C} 1-\mathrm{OH}$ in e.g. cellooligosaccharides ${ }^{15}$ and lactose ${ }^{16}$, xylooligosaccharides ${ }^{17}$ as well as chitooligosaccharides ${ }^{18}$ to the corresponding lactones. Electrons derived from oligosaccharide oxidation reduce the FAD cofactor that is subsequently re-oxidised via electron transfer to $\mathrm{O}_{2}$ to generate $\mathrm{H}_{2} \mathrm{O}_{2}$ (oxidase activity). Notably, comparable dehydrogenase and oxidase activities have been observed for an AA7 enzyme via electron transfer to artificial electron acceptors instead of $\mathrm{O}_{2}{ }^{16}$.

The tertiary structures of hitherto described AA7 enzymes ${ }^{15-17,19}$ share a common fold comprising an N-terminal FAD-binding domain (F domain) and a C-terminal substrate-binding domain (S domain). This fold is common within the vanillyl alcohol oxidase (VAO, EC. 1.1.3.38) super family ${ }^{20}$ that harbours AA7. All previously characterised AA7 oxidases are distinguished by a cysteinyl and histidyl bi-covalently tethered FAD cofactor. By contrast, other VAO family members harbour mostly a monocovalently (or less commonly a non-covalently) bound FAD cofactor $^{20}$.

Reactive oxygen species (ROS), and especially $\mathrm{H}_{2} \mathrm{O}_{2}$, play important roles in lignocellulose degradation by fungi, but the underpinning molecular details of these roles remain poorly understood ${ }^{11}$. Although $\mathrm{O}_{2}$ has long been considered as the cosubstrate of LPMOs ${ }^{5,7}$, recent findings suggest that $\mathrm{H}_{2} \mathrm{O}_{2}$ is the more favourable co-substrate during polysaccharide oxidative cleavage ${ }^{21-23}$. LPMO catalysis is mediated by a $\mathrm{Cu}$ cofactor $^{6}$ that must be reduced from $\mathrm{Cu}(\mathrm{II})$ to $\mathrm{Cu}(\mathrm{I})$ to prime the reaction. Enzymatic priming of LPMOs by the modular pyrroloquinolinequinone-dependent pyranose dehydrogenase ${ }^{24}$ (CAZy family AA12 dehydrogenase domain appended to an AA8 cytochrome $b$ haem domain) and FAD-dependant glucose-methanol-choline (GMC) superfamily of oxidoreductases (AA3), most notably the fungal cellobiose dehydrogenase $(\mathrm{CDH})$ that also possesses a cytochrome $b$ haem domain ${ }^{25-27}$, has been reported. The activity on cellooligosaccharides and the transcriptional co-regulation as well as the co-secretion with cellulose active AA9 $\mathrm{LPMOs}^{28}$, justified extensive studies on $\mathrm{CDH}$ as a model for direct electron transfer and priming of LPMOs ${ }^{25,27,29}$. Not all fungi possess the $\mathrm{CDH} / \mathrm{LPMO}$ pair, suggesting the presence of additional redox partners and mechanisms for LPMO activation. By analogy, the co-secretion of AA7s with LPMOs upon fungal growth on plant biomass $^{30,31}$ prompted us to hypothesize a redox interplay between these two enzyme classes.

Here, we report phylogenetic analyses, combined with the selection and characterisation of five fungal AA7s, three of which belong to previously unexplored clades. We demonstrate activity on four saccharides, previously not reported as AA7 substrates. Indepth analysis of a cellooligosaccharide dehydrogenase with a mono histidyl-tethered FAD highlights clade-dependant redox-profiles within AA7. Importantly, we unveil direct activation and potentiation of LPMO activity on cellulose by this newly-discovered dehydrogenase. This study provides biochemical, structural and mechanistic insights into AA7 enzymes as components of the fungal redox network secreted during growth on biomass. Our findings suggest a way to tune LPMO activity for enzymatic degradation of major recalcitrant polysaccharides using AA7 dehydrogenases.

\section{Results}

Clade-dependant variations in the FAD covalent tethering residues in AA7. To date, the molecular specificity signatures in AA7s have not been unveiled. To explore the sequence diversity in this family, we used the sequence of the previously characterised Fusarium graminearum chitooligosaccharide oxidase $F g \mathrm{ChitO}^{18}$ as a query to retrieve sequences comprising $470-570$ amino acids (aa) from a BLAST search. The sequences $(n=$ 1927), originating from fungi, eukaryotic microorganisms and plants, were aligned and curated to generate a phylogenetic tree formed by six clades (Fig. 1a). Clade I, which is the largest (34\% of all sequences), is dominated by plant and fungal sequences (Fig. 1b). Indeed, this clade contains plant non-carbohydrate active enzymes, e.g., the berberine bridge enzyme from Eschscholzia californica $E c \mathrm{BBE}^{32}$ and the monolignol oxidase from Arabidopsis thaliana AtBBE-like $15^{33}$, as well as the oligogalacturonide oxidase from $A$. thaliana $A t O G O X 1^{13}$, although none of these sequences are currently assigned into AA7.

The sequences in clades IIa, III and V are mainly from Ascomycota, whereas mostly Basidiomycota sequences populate clades IV and VI. Remarkably, the majority of the retrieved Ascomycota sequences were from genera known to harbour phytopathogens, e.g., Fusarium, Magnaporthe, Colletotrichum, Bipolaris, Alternaria or Botrytis. The enrichment of phytopathogen sequences is striking in clade II. Thus, fungus-like eukaryotic plant pathogens from Oomycota clustered in clade IIb $(90.5 \%$ of sequences), whereas the remaining sequences in clade II originate mainly from Ascomycota phytopathogens. All the four previously described AA7 fungal oligosaccharide oxidases clustered in a branch of clade V (Va) (Fig. 1a). The scarce insight into AA7 is evident from the lack of characterized members from (sub)clades II, III, IV, $\mathrm{Vb}$ and VI.

Since bi-covalent FAD tethering has been the hallmark of all hitherto characterised AA7s, we analysed the conservation of the cysteine and histidine FAD-tethering residues across the phylogenetic tree. Strikingly, clade II harboured exclusively non-canonical sequences with substitution of the FAD-binding cysteine, histidine or both, while these residues were highly conserved in the other clades (Fig. 1c). We selected five AA7 candidates belonging to different clades (Fig. 1a, Supplementary Table 1) for recombinant expression and functional characterization.

Identification of a strict oligosaccharide dehydrogenase in AA7. The selected fungal sequences share $25-39 \%$ amino acid sequence identity and originate from Aspergillus nidulans (AnAA7A, clade I), Fusarium graminearum ( $F g$ CelDH7C, clade IIa and $\mathrm{FgChi7B}$, clade $\mathrm{Va}$ ), Magnaporthe oryzae (MoChi7A, clade $\mathrm{Vb}$ ) and Polyporus brumalis (PbChi7A, clade VI) (Supplementary Table 1). The selected AA7 enzymes were successfully expressed in Pichia pastoris and highly pure enzymes were obtained from a single affinity purification. The substrate specificity of each recombinant enzyme was assessed against a panel of 40 compounds including saccharides with a degree of polymerization (DP) $1-4$ as well as sugar alcohols and aromatic alcohols (Supplementary Table 2) by monitoring both their oxidase activity (i.e. $\mathrm{H}_{2} \mathrm{O}_{2}$ production using a peroxidase coupled assay) and dehydrogenase 
a

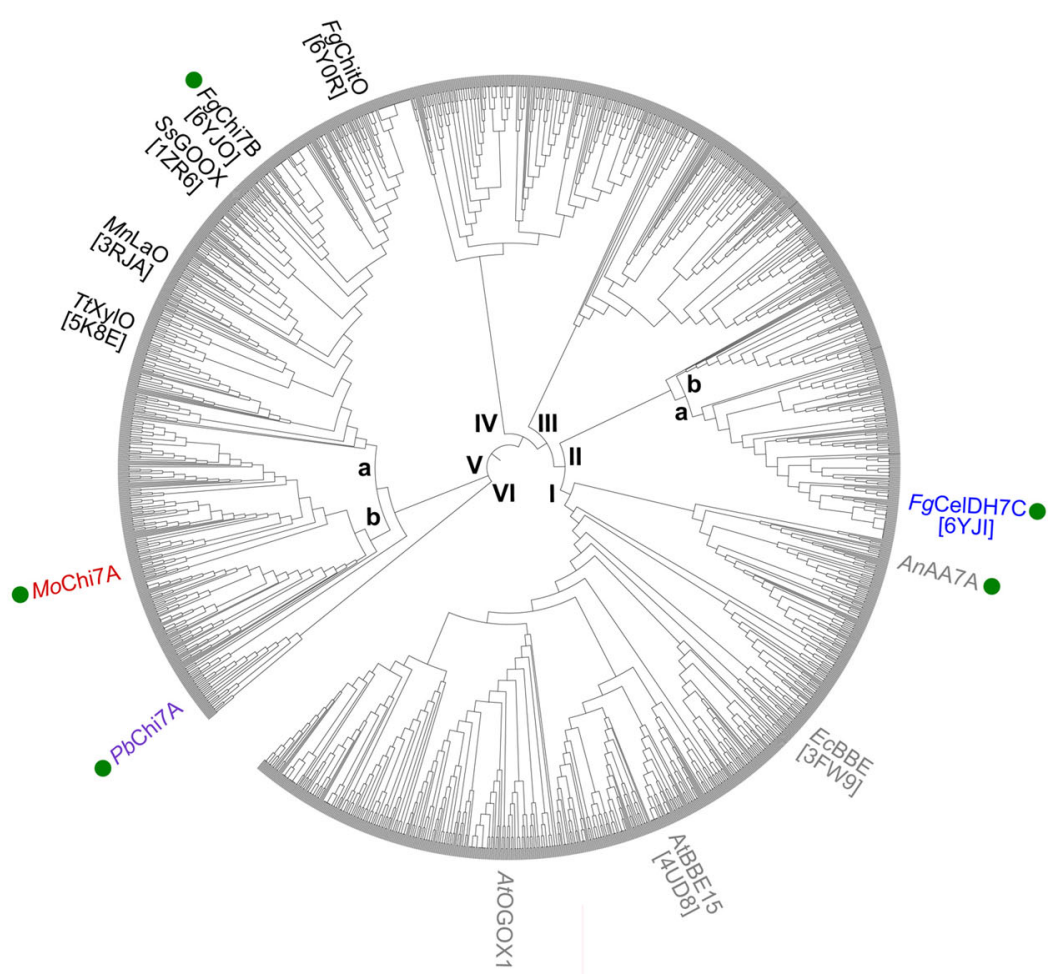

b
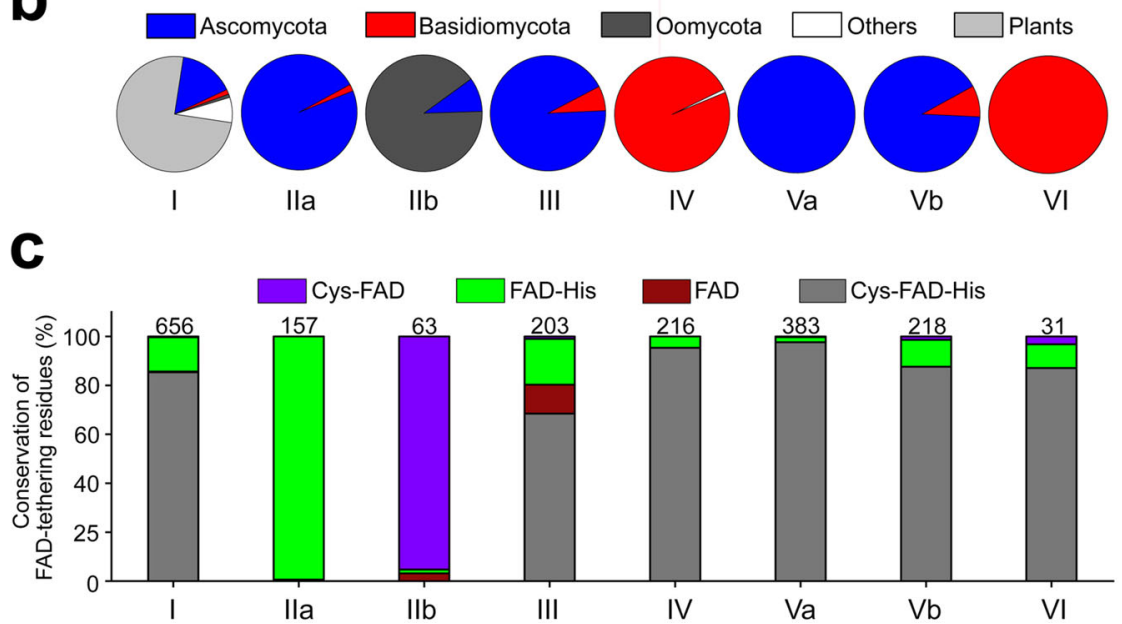

Fig. 1 Phylogenetic analysis of AA7-like sequences. a The phylogenetic tree is based on 1927 sequences. Biochemically characterized enzymes are coloured according to clade, with green circles indicating enzymes from the present study. The PDB entries (in square brackets) are given for available enzyme structures. Clade Va harbours the canonical previously described oligosaccharide oxidases: SsGOOX ${ }^{15}$ from Sarocladium strictum active on cellooligosaccharides, MnLaO ${ }^{16}$ from Microdochium nivale active on lactose, TtXylO'17 from Thermothelomyces thermophilus active on xylooligosaccharides and $\mathrm{FgChitO}^{18}$ from Fusarium graminearum active on chitooligosaccharides. Clade I contains characterised plant enzymes ${ }^{33,39}$ that are not assigned in AA7 including the oligogalacturonide oxidase $(A t O G O X 1)^{13}$ from Arabidopsis thaliana ${ }^{13,33,39}$. b Clade-wise taxonomic distribution of putative AA7 sequences. c Clade-wise conservation percentage of the histidine and cysteine FAD-cofactor tethering residues. The number of sequences within each clade is indicated above each bar. The accessions of the sequences in the tree are provided in the Source Data file.

activity using 2,6-dichlorophenolindophenol (DCIP) as an artificial electron acceptor.

FgChi7B (clade Va), MoChi7A (clade $\mathrm{Vb}$ ) and $\mathrm{PbChi7A}$ (clade $\mathrm{VI})$ displayed the highest normalised rates $\left(V_{\mathrm{o}} / \mathrm{E}\right)$ on chitooligosaccharides, followed by $\mathrm{N}$-acetyl glucosamine (GlcNAc) (Supplementary Fig. 1). Importantly, we also discovered oxidase activity against substrates not reported before in AA7. Thus, $\mathrm{MoChi7A}$ was active on $\mathrm{N}$-acetyl galactosamine (GalNAc), lacto$\mathrm{N}$-biose (LNB) and galacto- $\mathrm{N}$-biose (GNB), while PbChi7A oxidised mannooligosaccharides (Supplementary Table 3, Supplementary Fig. 1). The $K_{\mathrm{M}}$ values of $M o$ Chi7A and $\mathrm{PbChi7 \textrm {A }}$ towards GlcNAc were about 20 -fold lower compared to $\mathrm{FgChi7B}$ (Table 1), highlighting a marked difference in affinity for monosaccharides between these enzymes. No activity was detected for AnAA7A on any of the tested substrates, suggesting that AA7 targets a wider range of substrates than currently reported. The $\mathrm{pH}$-dependence of activity profiles showed the highest relative activities in the $\mathrm{pH}$ range 7.0-7.5, except for

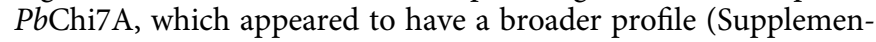
tary Fig. 2).

Apart from $\mathrm{FgCelDH7C} \mathrm{(vide} \mathrm{infra),} \mathrm{the} \mathrm{dehydrogenase}$ activity for a given substrate was in the same range or higher 


\section{Table 1 Apparent kinetic parameters of AA7-catalysed} oxidation of saccharides.

Oxidase kinetics

\begin{tabular}{|c|c|c|c|c|}
\hline \multirow[t]{2}{*}{ Substrate } & \multirow[t]{2}{*}{ Parameter } & \multicolumn{3}{|l|}{ Enzyme } \\
\hline & & MoChi7A & PbChi7A & FgChi7B \\
\hline \multirow[t]{2}{*}{ GalNAc } & $k_{\text {cat }}\left(s^{-1}\right)$ & $3.7 \pm 0.1$ & ND & ND \\
\hline & $K_{M}(\mathrm{mM})$ & $\begin{array}{l}1.69 \pm 0.12 \\
2.16 \times 10^{3}\end{array}$ & ND & ND \\
\hline \multirow[t]{4}{*}{ GlcNAc } & $k_{\text {cat }}\left(s^{-1}\right)$ & $\begin{array}{l}2.16 \times 10 \\
3.8 \pm 0.1\end{array}$ & $\begin{array}{l}3.0 \pm 0.2 \\
3.3 \pm 0.2^{a}\end{array}$ & $6.8 \pm 0.3$ \\
\hline & $K_{M}(\mathrm{mM})$ & $0.80 \pm 0.05$ & $0.69 \pm 0.20$ & $15.7 \pm 1.13$ \\
\hline & $k_{\text {cat }} / K_{M}\left(s^{-1} M^{-1}\right)$ & $4.75 \times 10^{3}$ & $\begin{array}{l}4.27 \times 10^{3} \\
5.94 \times 10^{3 a}\end{array}$ & $4.30 \times 10^{2}$ \\
\hline & $k_{\text {cat }}\left(s^{-1}\right)$ & $\begin{array}{l}4.4 \pm 0.1 \\
18.3 \pm 0.4^{a}\end{array}$ & $3.8 \pm 0.04$ & $\begin{array}{l}2.3 \pm 0.03 \\
28.7 \pm 0.4\end{array}$ \\
\hline \multirow[t]{3}{*}{ Chitobiose } & $K_{\mathrm{M}}(\mathrm{mM})$ & $0.064 \pm 0.01$ & $0.3 \pm 0.02$ & $0.27 \pm 0.01$ \\
\hline & $k_{\text {cat }} / K_{M}\left(s^{-1} M^{-1}\right)$ & $\begin{array}{l}0.33 \pm 0.03^{\mathrm{a}} \\
6.83 \times 10^{4} \\
5.56 \times 10^{4 a}\end{array}$ & $1.27 \times 10^{4}$ & $\begin{array}{l}1.32 \pm 0.07 \\
8.58 \times 10^{3} \\
217 \times 10^{4 a}\end{array}$ \\
\hline & $k_{\text {cat }}\left(s^{-1}\right)$ & $3.71 \pm 0.09$ & $\begin{array}{l}3.55 \pm 0.04 \\
2.0 \pm 0.1^{\mathrm{a}}\end{array}$ & $6.14 \pm 0.29$ \\
\hline \multirow[t]{2}{*}{ Chitotriose } & $K_{\mathrm{M}}(\mathrm{mM})$ & $0.09 \pm 0.01$ & $0.28 \pm 0.01$ & $0.25 \pm 0.03$ \\
\hline & $k_{\text {cat }} / K_{M}\left(s^{-1} M^{-1}\right)$ & $4.22 \times 10^{4}$ & $\begin{array}{l}1.25 \times 10^{4} \\
2.67 \times 10^{3 a}\end{array}$ & $2.44 \times 10^{4}$ \\
\hline
\end{tabular}

Dehydrogenase kinetics of FgCelDH7C.

\begin{tabular}{lllll}
\hline & Cellobiose & Cellotriose & Cellotetraose & Cellopentaose \\
\hline$k_{\text {cat }}\left(\mathrm{s}^{-1}\right)$ & $30.8 \pm 0.81$ & $36.1 \pm 0.74$ & $39.27 \pm 0.76$ & $45.28 \pm 2.03$ \\
$K_{M}(\mathrm{mM})$ & $5.35 \pm 0.39$ & $3.94 \pm 0.22$ & $6.06 \pm 0.27$ & $11.25 \pm 0.94$ \\
$k_{\text {cat }} / K_{M}$ & 5757 & 9162 & 6480 & 4025 \\
$\left(s^{-1} \mathrm{M}^{-1}\right)$ & & & & \\
\hline
\end{tabular}

aApparent kinetic parameters using the dehydrogenase assay. The kinetic parameters are determined from a global fit of the Michaelis-Menten expression to triplicate ( $n=3$ independent experiments), except for cellopentaose ( $n=1$ experiment). The data are shown as means \pm standard deviations. For cellopentaose the error estimates of the fit to the single data set are shown.

(up to 12 -fold) than the corresponding oxidase activity, and the substrate specificity profiles were mostly similar using both assays (Table 1, Supplementary Fig. 1). By contrast, FgCelDH7C acted as a dehydrogenase with a preference for cellooligosaccharides with $k_{\text {cat }}$ values of $32-42 \mathrm{~s}^{-1}$ and the highest catalytic efficiency on cellotriose (Table 1, Supplementary Fig. 1h). The enzyme was also active on glucose, $\alpha$-(1,4)-glucooligomers (maltooligosaccharide) and lactose, all sharing a reducing end glucosyl unit. Interestingly, the oxidase activity was estimated to be several orders of magnitude lower $\left(V_{\mathrm{o}} / \mathrm{E} \approx 1-10 \times 10^{-5} \mathrm{~s}^{-1}\right) \quad$ (Supplementary Fig. $1 \mathrm{~g}, \mathrm{~h}$ ). This uniquely higher dehydrogenase/oxidase activity ratio compared to canonical AA7s distinguishes $\mathrm{FgCelDH7C}$ as an AA7 dehydrogenase with only trace oxidase activity. Interestingly, FgCelDH7C had a markedly different flavin absorbance spectrum than typical oxidase homologues (Fig. 2a), suggestive of large changes in the FAD chemical environment. The stability of the enzyme was also evaluated to verify the structural integrity (unfolding temperature $T_{\mathrm{m}}>55^{\circ} \mathrm{C}$, Supplementary Fig. 2b). Both MoChi7A, which catalyses efficient oxidation of substrates not reported within AA7, and the strict dehydrogenase $\mathrm{FgCelDH7C}$ were selected for further in situ NMR spectroscopy to bring insight into their redox chemistry. Initial experiments identified the chemical shift assignments of substrates, intermediates and products based on two-dimensional ${ }^{1} \mathrm{H}-{ }^{13} \mathrm{C}$ NMR analyses (Supplementary Fig. 3). Time-resolved two-dimensional ${ }^{1} \mathrm{H}_{-}{ }^{13} \mathrm{C}$ HSQC NMR spectra were acquired to follow the time-course conversion of GlcNAc and GalNAc by $M o C h i 7 A$, showing the initial formation of a 1,5-pyrano $(\delta)$ lactone prior to rearrangement to a 1,4-furano $(\gamma)$ lactone. The carboxylic acid open form was the main product of GlcNAc oxidation (due to lactone hydrolysis), whereas the 1,4 -furano $(\gamma)$ lactone accumulated as the main product of GalNAc oxidation (Supplementary Fig. 4). Similarly, assignments were established for the oxidation of cellobiose by $\mathrm{FgCelDH7C} \mathrm{(Supplementary}$ Fig. 5). A time series of one-dimensional ${ }^{1} \mathrm{H}$ NMR spectra was used for kinetic analysis of the FgCelDH7C catalysed oxidation of cellobiose in the presence of DCIP that displayed exchange line broadening during the reaction (Supplementary Fig. 6a). Line shape analysis showed that oxidised DCIP was reduced in a slow to intermediate exchange regime (exchange rate $\approx 87 \mathrm{~s}^{-1}$ ) (Supplementary Fig. 6b). Interestingly, the sharp signals of reduced DCIP were only observed upon stoichiometric conversion of the oxidized DCIP form and hydrolysis of the lactone to the acid (Supplementary Fig. 6a), most likely due to the irreversible hydrolysis breaking the equilibrium. Notably, the oxidase reaction with $\mathrm{O}_{2}$ as an electron acceptor occurred orders of magnitude slower in the absence of DCIP as electron acceptor (Fig. 2b, c), corroborating the activity data from the coupled peroxidase assay above. Further NMR analyses and activity assays validated the dependence of cellooligosaccharide oxidation on DCIP concentration (Supplementary Fig. 7).

Structural elements underpinning the dehydrogenase/oxidase activity profiles. We determined the crystal structures of $\mathrm{FgChi7B}$ and $\mathrm{FgCelDH7C}$ at resolutions of 2.4 and $1.6 \AA$, respectively (Supplementary Table 4, Supplementary Fig. 8). Both enzymes share the canonical AA7 fold comprising an FAD-binding F domain and a substrate-binding $S$ domain that is formed by a central $\beta$-sheet flanked by $\alpha$-helices (Supplementary Fig. 9a, b). Moreover, the two enzymes share an aromatic cluster comprising: (i) the catalytic base tyrosine (FgCelDH7C Y454), (ii) a tyrosine/ phenylalanine ( $F g C e l D H 7 C$ F99) that stacks onto and stabilises the catalytic base, and (iii) an aromatic residue that stacks mainly onto the saccharide unit penultimate to the reducing end (FgCelDH7C F383) (Fig. 3a). Interestingly, the residues of this aromatic cluster are also highly conserved in most sequences in clades IIa and Va (Fig. 3b), highlighting their importance in catalysis and substrate binding.

By contrast, the structure of $\mathrm{FgCelDH7C}$ revealed a unique active site architecture, as compared to all hitherto available AA7 structures. The first notable overall structural difference is that a loop (residues Q335-F346) that flanks the active site in $\mathrm{FgCelDH7C}$ appears shorter due to a preceding helical segment that folds away from the active site, which provides a less occluded and more solvent-exposed FAD-cofactor compared to FgChi7B and all hitherto reported AA7 structures (Supplementary Fig. 9).

Strikingly, the mode of FAD-anchoring represents a second unique characteristic distinguishing $\mathrm{FgCelDH} 7 \mathrm{C}$ from the other AA7 structures. Thus, the isoalloxazine ring of the FAD cofactor in $\mathrm{FgChi7B}$ is bi-covalently anchored at the $\mathrm{C} 6$ atom to $\mathrm{C} 162 \mathrm{~S} \gamma$ (6-S-cysteinyl) and the $8 \alpha$-methyl group to $\mathrm{H} 102 \mathrm{~N}^{\delta 1}(8 \alpha-N 1$ histidyl), akin to all previously determined AA7 structures (Supplementary Fig. 9a). By contrast, FgCelDH7C is monocovalently histidyl-tethered due to the substitution of the FADtethering cysteine to a glycine (Fig. 3c). Notably, this cysteine is conserved in the majority of AA7 except in clade II sequences that possess a glycine, an alanine or a serine at this position (Fig. 3b, d, Supplementary Fig. 10). A third structural signature of FgCelDH7C, compared to all available AA7 structures, is the substitution of a histidine residue facing the isoalloxazine ring in the FAD-binding domain to a serine (S165) (Fig. 3c).

A fourth different motif in $\mathrm{FgCelDH7C}$ entails four arginine residues that protrude into the substrate-binding pocket (Fig. 3c, d), providing a distinctively positive charged milieu compared to other structurally characterised AA7s (Supplementary Fig. 11). 


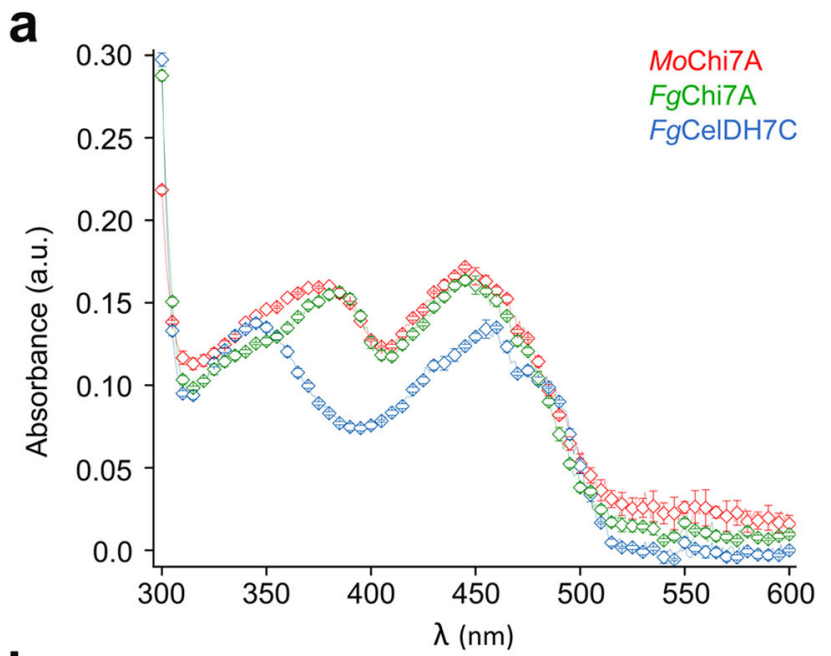

b

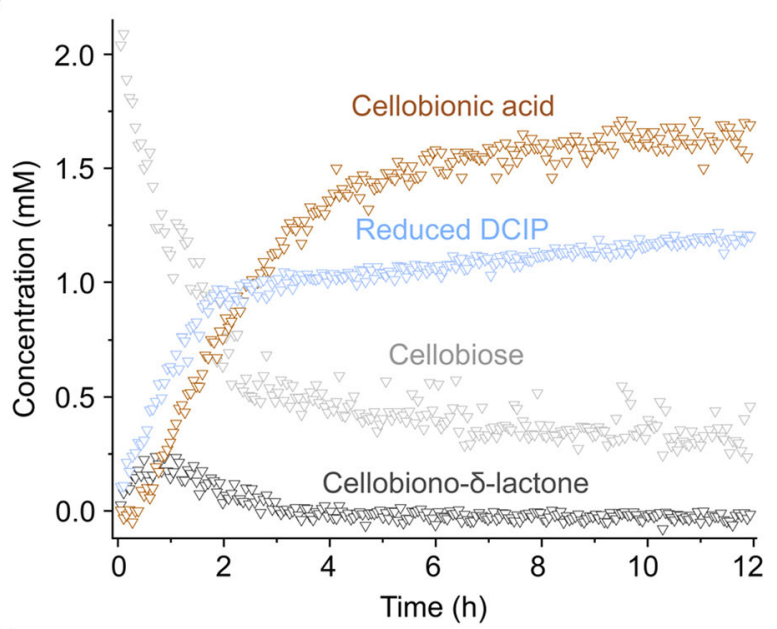

C

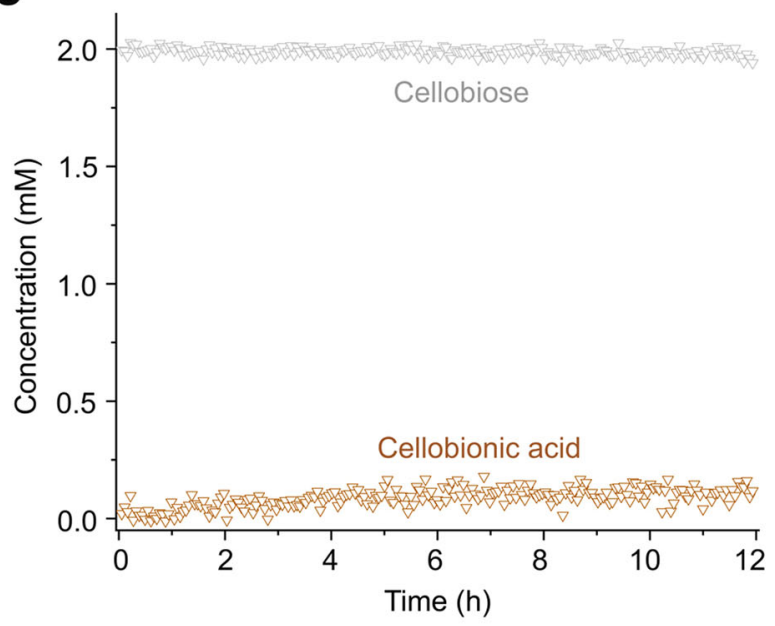

Fig. 2 Spectral properties and time-course NMR analysis of the AA7 cellooligosaccharide dehydrogenase FgCeIDH7C. a Spectral comparison of the flavin absorbance of $\mathrm{FgCeIDH7C}$ and the oxidases $\mathrm{FgChi7B}$ as well as MoChi7A, all at $20 \mu \mathrm{M}$. The data are means \pm standard deviations $(n=3$ independent experiments). $\mathbf{b}$ and $\mathbf{c}$ Show the time resolved in situ ${ }^{1} \mathrm{H}$ NMR analyses of the oxidation of cellobiose by $\mathrm{FgCeIDH7C}$ in the presence (b) or absence (c) of $1.3 \mathrm{mM} \mathrm{2,6-dichlorophenolindophenol} \mathrm{(DCIP)} \mathrm{as} \mathrm{an}$ electron acceptor, respectively. The presented spectra in panels $b$ and $c$ are from a single experiment $(n=1)$. Source data are provided as a Source Data file.
Notably, members of clade IIb share the above signatures with clade IIa, except for the inverted substitution of the FADtethering residues. Hence, the cysteine is conserved, but the histidine counterpart is substituted for an arginine (Fig. 1, Supplementary Fig. 10). In conclusion, the present analysis allows the identification of active-site signatures associated with the functional variation in AA7, e.g., activity on oligosaccharides and the dehydrogenase versus the typical oxidase activity.

The cellooligosaccharide dehydrogenase $\mathrm{FgCelDH7C}$ primes and fuels LPMO activity. To investigate if AA7 enzymes can trigger the activity of LPMOs, we set up AA7-LPMO mediated cellulose degradation assays to monitor the release of native (unmodified) and oxidised cellooligosaccharides from cellulose. The two cellulose-active LPMOs from Podospora anserina $\mathrm{PaLPMO} 9 \mathrm{E}$ and $\mathrm{PaLPMO} 9 \mathrm{H}^{34}$ were used in these assays. The interplay between $\mathrm{FgCelDH7C}$ and the C1-oxidising PaLPMO9E was evident from the marked increase in native and $\mathrm{C} 1$-oxidised cellooligosaccharides released from phosphoric acid swollen cellulose (PASC) relative to the control reaction, which was fuelled by electrons from ascorbate (Supplementary Fig. 12a). Since $\mathrm{FgCelDH7C}$ is inactive on cellulose, the release of oligosaccharides in this assay is solely attributed to the PaLPMO9E activity. However, the generation of $\mathrm{C} 1$-oxidised cellooligosaccharides, by both $F g C e l D H 7 C$ and PaLPMO9E, precluded determining the contribution of each enzyme to the total amount of oxidized species. Therefore, a similar assay was also performed with the C4-oxidising $\mathrm{PaLPMO} 9 \mathrm{H}$ (instead of $\mathrm{PaLPMO} 9 \mathrm{E}$ ) as it allowed attributing both $\mathrm{C} 4$ and $\mathrm{C} 1 / \mathrm{C} 4$ oxidised cellooligosaccharides (identification based on the previous work ${ }^{34}$ ) exclusively to the LPMO activity. This assay with $\mathrm{PaLPMO} 9 \mathrm{H}$ resulted in a significant increase in single- and double-oxidized oligosaccharides relative to the ascorbate control (Supplementary Fig. 12b). We performed similar assays on Avicel (higher crystallinity cellulose than PASC), which revealed that the addition of the preferred $\mathrm{FgCelDH7C}$ substrate cellotetraose (DP4) results in a considerable increase in the level of released cellooligosaccharides in a dose-dependent manner (Supplementary Fig. 13). A similar fuelling of LPMO activity was also observed when the same assay was performed using the previously characterised C4-oxidising AA9 LPMO from Lentinus similis (LsAA9A) ${ }^{35,36}$ (Supplementary Fig. 14). These findings indicate that the ability of $\mathrm{FgCelDH7C}$ to fuel cellulose active LPMOs is not LPMO-specific.

We further used the $\mathrm{CDH}$ from $P$. anserina to benchmark the $\mathrm{FgCelDH7C-PaLPMO9H}$ system, as $\mathrm{CDH}$ are recognised as key redox partners to AA9 $\mathrm{LPMOs}^{26,27,34}$. The observed release of cello-oligomers was markedly higher when the LPMO reaction was fuelled by a 3 -fold lower FgCelDH7C concentration compared to $\mathrm{PaCDHB}$ (Fig. 4, Supplementary Fig. 15). Indeed, the $\mathrm{FgCelDH7C-PaLPMO9H}$ pair released 1.5 - and 2-fold higher C1-C4 double-oxidised and native cellooligosaccharides, respectively, as compared to the $\mathrm{PaCDHB}$-fuelled reaction. To evaluate if this interplay was specific for $\mathrm{FgCelDH7C}$, we used the promiscuous oxidase $\mathrm{MoChi7 \textrm {A }}$ in a similar cellulose degradation assay. Interestingly, the high level of AA7-fuelled LPMO activity appeared specific to $\mathrm{FgCelDH7C}$ based on the low amounts of C1and C1-C4 oxidised species observed in assays where FgCelDH7C was replaced with MoChi7A that displays low cellooligosaccharides oxidase side-activity (Supplementary Fig. 16).

Mechanistic insights into the LPMO-AA7 interplay. To investigate the mechanism of the AA7-LPMO interplay, we analysed the effect of $\mathrm{H}_{2} \mathrm{O}_{2}$ or the superoxide species $\left(\mathrm{O}_{2}{ }^{\bullet-}\right)$ by performing the reaction in the presence of either horseradish peroxidase (HRP) that converts $\mathrm{H}_{2} \mathrm{O}_{2}$ to $\mathrm{H}_{2} \mathrm{O}^{21}$ or superoxide dismutase 


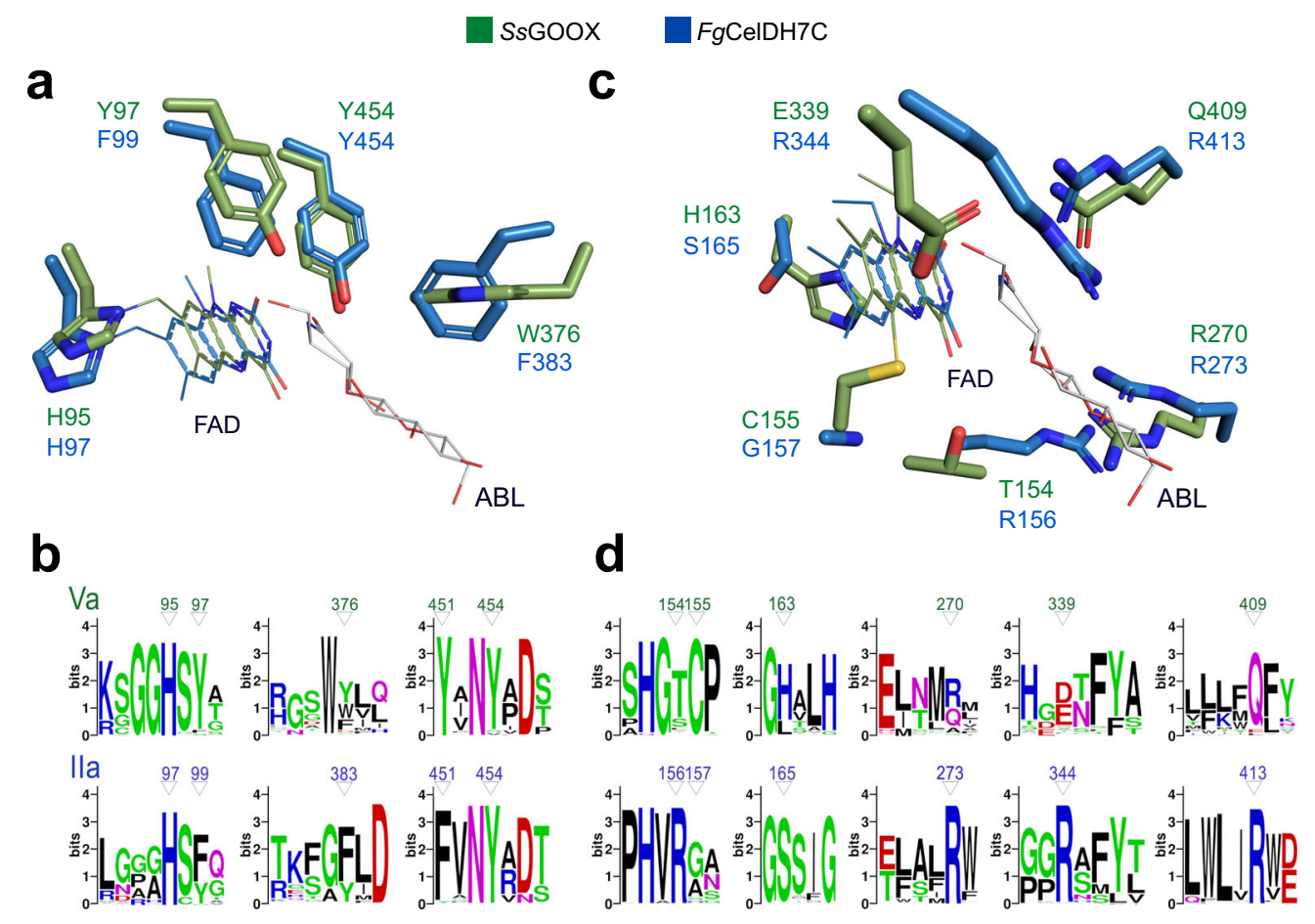

Fig. 3 Active site signatures of AA7 oligosaccharide oxidases versus dehydrogenases. The active sites of the clade lla discovered dehydrogenase FgCeIDH7C (PDB: 6YJI) and the canonical clade Va cellooligosaccharide oxidase SsGOOX (in complex with 5-amino-5-deoxy-cellobiono-1,5-lactam ABL, PDB: $2 A X R$ ) are shown. a The FAD-tethering histidine in addition to an aromatic cluster comprising the tyrosine base catalyst, a phenylalanine/tyrosine and the substrate-stacking aromatic residue are conserved features in fungal oligosaccharide dehydrogenases and oxidases. $\mathbf{b}$ Sequence logos of patches spanning the structurally similar active site residues shown in (a). c Active site differences between clades Ila and Va. $\mathbf{d}$ Sequence logos of patches spanning the structurally divergent active site residues shown in c from clades Ila and $\mathrm{Va}$. The amino acid numbering of the deposited protein sequences is used in the figure.
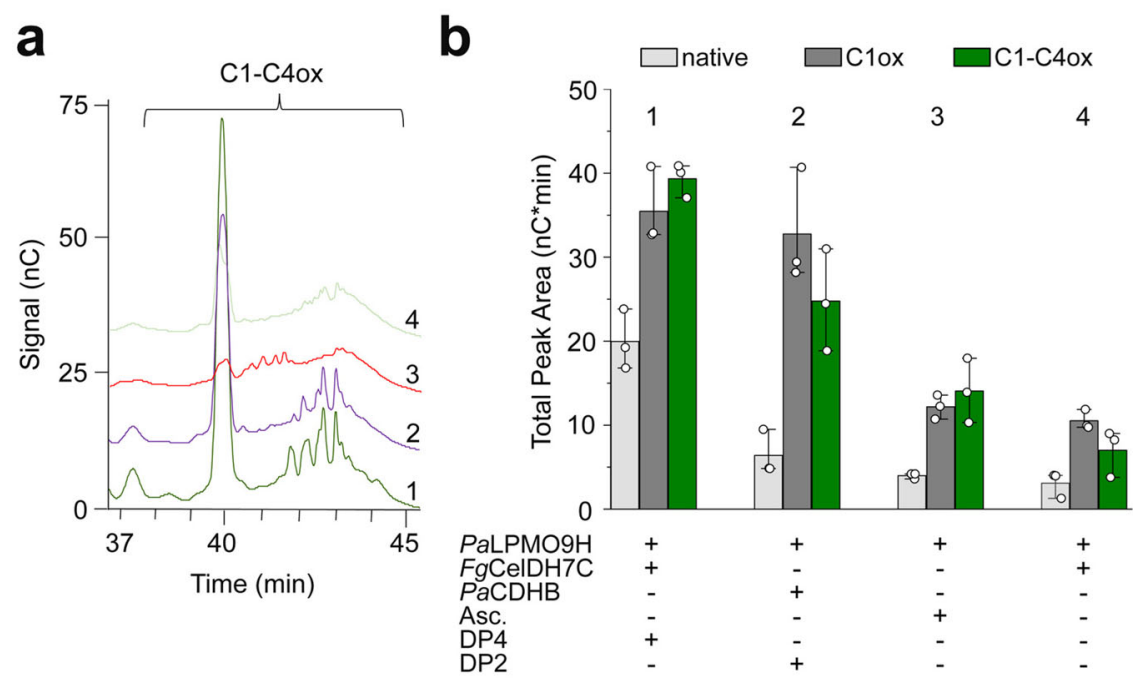

Fig. 4 Analysis of AA7-LPMO interplay in cellulose degradation. Reactions were performed on Avicel using FgCelDH7C and PaLPMO9H with subsequent ionic chromatography (HPAEC-PAD) analysis. a A representative chromatogram part showing C1-C4 double-oxidised species in the cellulose degradation assay including combinations of Avicel $\left(5 \mathrm{mg} \mathrm{mL}^{-1}\right), \mathrm{FgCeIDH7C}(0.4 \mu \mathrm{M}), \mathrm{PaLPMO} 9 \mathrm{H}(4 \mu \mathrm{M}), \mathrm{PaCDHB}(1.2 \mu \mathrm{M})$, cellotetraose (DP4, $\left.0.8 \mathrm{mM}\right)$ and ascorbate (Asc., $1 \mathrm{mM}$ ) as indicated in the figure. $\mathbf{b}$ Comparison of the cellulose degradation assay based on the cumulative area under the peaks of native (DP3, DP5 and DP6), C1 oxidised (except DP2 and DP4 which were added as substrates for the CDH and AA7, respectively) and C1-C4 double-oxidised cellooligosaccharides from the reactions in (a). The data in (a) and (b) ( $n=3$ independent reactions) were generated in $\mathrm{NaOAc} / \mathrm{NaOH}$ buffer ( $50 \mathrm{mM}$, $\mathrm{pH}$ 5.2) at $35^{\circ} \mathrm{C}$. The bar plot in (b) shows the means of total peak area ( $n=3$ independent reactions, each shown as a white circle) with standard deviations. Source data are provided as a Source Data file. 

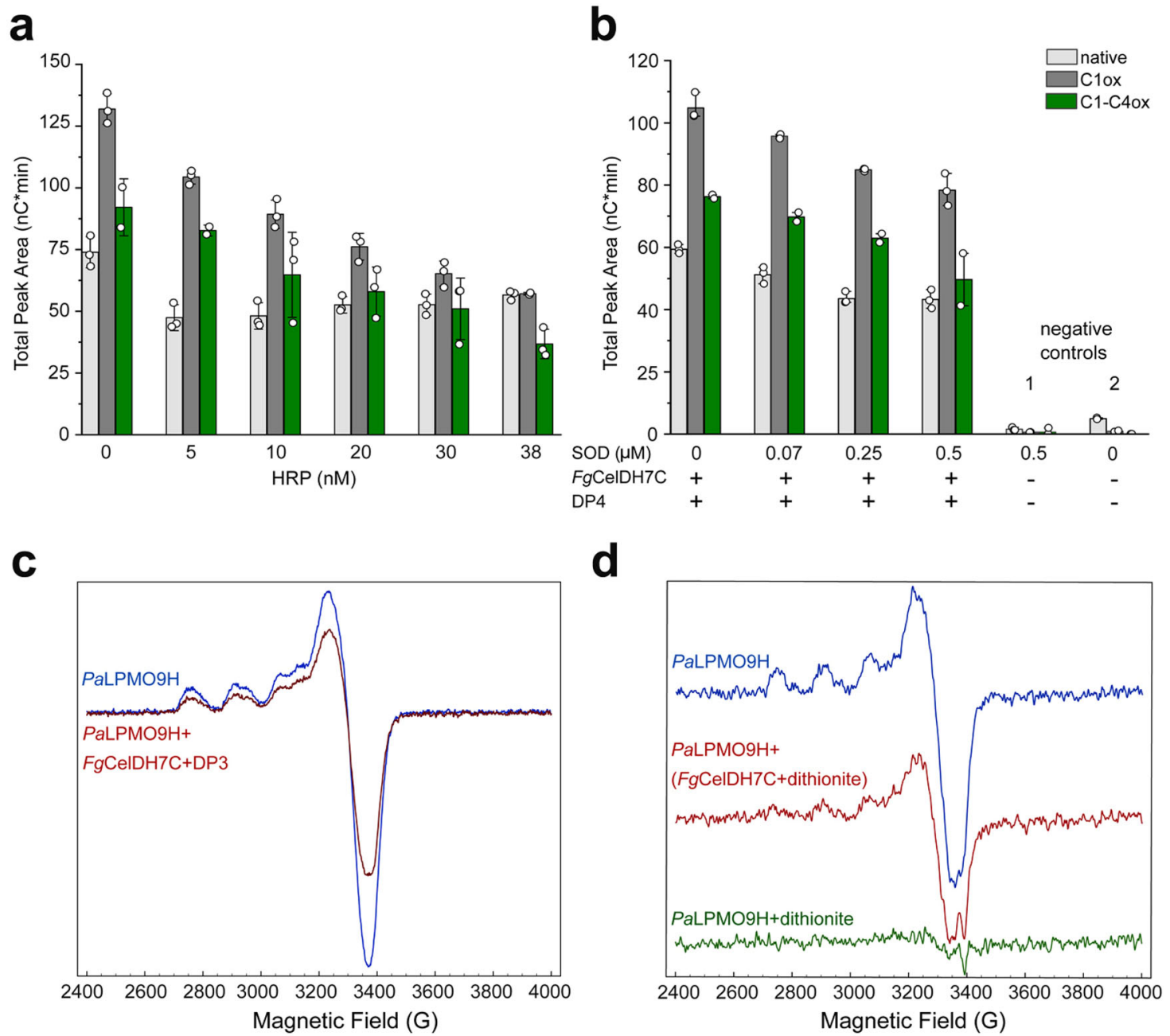

Fig. 5 Mechanistic insights into the activation of LPMO by FgCelDH7C. a, b Effect of horseradish peroxidase (HRP) and superoxide dismutase (SOD) on the interplay between $\mathrm{PaLPMO} 9 \mathrm{H}(4 \mu \mathrm{M})$ and $\mathrm{FgCelDH7C}(0.41 \mu \mathrm{M})$ in Avicel degradation based on the total area of native, $\mathrm{C} 1$ oxidised $(\mathrm{C} 1$ ox $)$ and double C1-C4 oxidised (C1-C4ox) cello-oligosaccharides as analysed by HPAEC-PAD. Controls prepared in the absence of FgCelDH7C (1) or in the absence of both SOD and FgCelDH7C (2). The assays were performed for $18 \mathrm{~h}$ at $35^{\circ} \mathrm{C}$ and terminated using $\mathrm{NaOH}(0.1 \mathrm{M})$ prior to the HPAEC-PAD analyses. The total peak area (white circles, $n=3$ independent experiments) are shown and the bar plots display the means with standard deviations. $\mathbf{C}$ band Electron Paramagnetic Resonance (EPR) spectra of PaLPMO9H-Cu(II) $(100 \mu \mathrm{M})$ in the presence of cellotriose (DP3, $1 \mathrm{mM})$ before (blue line) and after (red line) addition of FgCeIDH7C (AA7). d X-band EPR spectra of PaLPMO9H-Cu(II) $(20 \mu \mathrm{M})$ in buffer (blue line), in the presence of FgCelDH7C (AA7) pre-reduced with dithionite (red line), or directly fully reduced with dithionite (10 eq., green line). All EPR solutions and experiments were performed under anaerobic conditions. Samples were in $50 \mathrm{mM} \mathrm{NaOAc}$, pH 5.2 and spectra were recorded at $50 \mathrm{~K}$ with a $4 \mathrm{~mW}$ microwave power and a $30 \mathrm{Gauss}$ modulation amplitude. The data in $(\mathbf{c})$ and $(\mathbf{d})$ are based on a single experiment $(n=1)$. Source data for the a and b panels are provided as a Source Data file.

(SOD) that converts superoxide ions ${ }^{37}$ to $\mathrm{H}_{2} \mathrm{O}_{2}$ and $\mathrm{O}_{2}$, respectively. The addition of increasing concentrations $(5-38 \mathrm{nM})$ of HRP, reduced the AA7-potentiated LPMO activity (Fig. 5a). These results are consistent with $\mathrm{H}_{2} \mathrm{O}_{2}$, which is supplied by FgCelDH7C and by the side-activity of primed LPMOs, being a preferred LPMO co-substrate. In the presence of increasing concentrations $(0.07-0.5 \mu \mathrm{M})$ of SOD $^{37}$, we observed a similar inhibitory trend (Fig. 5b). These findings suggest that $\mathrm{O}_{2}{ }^{--}$plays a specific role in the FgCelDH7C-fuelled LPMO reaction (Fig. 5b). Indeed, the priming reduction of LPMOs with the superoxide ion has been previously proposed ${ }^{38}$. In addition to $\mathrm{O}_{2}{ }^{\bullet-}$-mediated priming, we also investigated the possibility of direct priming electron transfer between the AA7 and the LPMO using Electron Paramagnetic Resonance (EPR) spectroscopy. The extent of LPMO-Cu(II) reduction to $\mathrm{Cu}(\mathrm{I})$ was monitored as a decrease of the $\mathrm{Cu}$ (II) EPR signal due to the silence of LPMO-Cu(I) species in $\mathrm{EPR}^{25}$. The addition of $\mathrm{FgCelDH7C}$ to $\mathrm{PaLPMO} 9 \mathrm{H}$ pre-incubated with cellotriose (DP3) under anaerobic conditions led to a decrease (35-40\%) in PaLPMO9H-Cu(II) signal (Fig. 5c), whereas DP3 alone failed to induce a change in the $\mathrm{Cu}$ (II) EPR signal intensity, consistent with the involvement of $\mathrm{FgCelDH7C}$ in the observed priming of $\mathrm{PaLPMO} 9 \mathrm{H}$ (Fig. 5d). The fact that only partial reduction of the $\mathrm{Cu}$ (II) center was observed could be due to kinetic or thermodynamic barriers. We have previously determined the redox potentials of the LPMOs $P a A A 9 E$ and $P a A A 9 H$ at $\mathrm{pH} 5$ to $+155 \mathrm{mV}$ and $+326 \mathrm{mV}$, respectivley ${ }^{25}$. The redox potentials of canonical AA7 oxidases with a bi-covalently anchored-FAD is about $+130 \mathrm{mV}$, whereas mutants that abolish the cysteinyl-FAD bond exhibited redox potentials in the $60 \mathrm{mV}$ range ${ }^{39,40}$. Accordingly, the reduction of LPMOs by FgCelDH7C is likely to be thermodynamically feasible assuming a redox potential in the same range as the abolished cysteinyl-FAD bond mutants. To test the possibility of a direct electron transfer between $\mathrm{FgCelDH7C}$ and $\mathrm{PaLPMO} 9 \mathrm{H}$, we used a strategy 
previously developed to analyse electron transfer between redox partner proteins ${ }^{41}$. We first established that $\mathrm{PaLPMO} 9 \mathrm{H}-\mathrm{Cu}$ (II) could be efficiently reduced by the chemical reductant dithionite (Fig. 5d). Then, equimolar solutions of FgCelDH7C and PaLP$\mathrm{MO} 9 \mathrm{H}$ were prepared anaerobically. The $\mathrm{FgCelDH7C}$ solution was pre-reduced with sub-stoichiometric (80\%) amounts of dithionite to avoid excess dithionite in the medium. Identical volumes of the two protein solutions were then mixed which resulted in about 50\% reduction of the LPMO active site from $\mathrm{Cu}$ (II) to the $\mathrm{Cu}(\mathrm{I})$ state (Fig. $5 \mathrm{~d}$ ). Altogether, our data are indicative of a molecular oxygen-independent, direct electron transfer from the AA7-bound FAD to the active site of the LPMO.

\section{Discussion}

This study sheds light on an enigmatic family of flavo-enzymes mainly occurring in fungi and fungi-like eukaryotic microorganisms as well as in plants. Our phylogenetic analysis mapped the four hitherto described AA7 fungal oligosaccharide oxidases together with a large set of mostly uncharacterised orthologues into six clades. The majority of clade I sequences share a highly conserved motif involving the catalytic tyrosine base and a stacking tyrosine (or phenylalanine) that is likely to rigidify the position of the catalytic base (Fig. 3a, Supplementary Fig. 10). Curiously, a few atypical enzymes lack the catalytic tyrosine, e.g., $E c B B E$ from clade I possess a histidine and an adjacent glutamic acid at the equivalent position in the structure. This is in agreement with an alternative mechanism, involving acid/base catalysis previously proposed for $E c \mathrm{BBE}^{42}$. Nonetheless, the conservation of the catalytic motif in most sequences supports the conservation of the canonical mechanism across all AA7 clades. These findings expand significantly the sequence inventory that can be considered as AA7.

The substrate aromatic-stacking platform (Phe, Tyr or Trp) (Fig. 3a, Supplementary Fig. 10), is conserved in all previously and presently described oligosaccharides-active AA7s, in addition to a clade I enzyme that is specific for aromatic alcohols ${ }^{33}$, indicative of shared substrate-stacking function of the above aromatic platform. By contrast, sequences that lack the aromaticstacking residue are unlikely to be active on oligosaccharides, e.g., $E c \mathrm{BBE}$, which features in alkaloid biosynthsis ${ }^{32}$. In conclusion, the above described aromatic-stacking platform offers a signature for the identification of putative carbohydrate-active AA7 members, which are likely to have more polar substrate-binding pockets than counterparts active on aromatic substrates. An active site arginine (FgCelDH7C R273) (Fig. 3c, d) has been previously proposed as a specificity signature of carbohydrateactive AA7 members ${ }^{18}$. This arginine correlates with activity on substrates that lack an $\mathrm{N}$-acetyl C2-substituent, e.g., cellooligosaccharides and xylo-oligosaccharides. A glutamine or smaller amino acids (Fig. 3d) at this position allow the accommodation of $\mathrm{N}$-acetylated substrates, e.g., chito-oligosaccharides consistent with our structural analysis of $\mathrm{FgChi7B}$ and the previously characterised $\mathrm{FgChitO}^{18,19}$ (Supplementary Fig. 17).

Interestingly, $\mathrm{FgCelDH7C}$ offers structural and functional insight into the previously unknown clade II (Fig. 3b, d), which is exclusively populated with atypical sequences of enzymes with potentially mono-histidyl (clade IIa), mono-cysteinyl or noncovalently bound FAD (clade IIb). The lower $(<10,000$-fold) oxidase activity of $\mathrm{FgCelDH7C}$ compared to canonical AA7s correlated with conspicuous changes in its active site architecture. The loss of cysteinyl-FAD is likely to elicit a large decrease in the midpoint redox potential, as observed in $\operatorname{EcBBE}^{39}$ (clade I) and $\mathrm{SsGOOX}^{40}$ (clade Va) when the cysteinyl bond is abolished ${ }^{39}$. In both cases, the redox potentials decreased from about $130 \mathrm{mV}$ to $53-61 \mathrm{mV}$ accompanied by about 20 -fold decrease in oxidase activity. The changes in the flavin absorbance spectrum due to the loss of the cysteinyl-bond in both enzymes were similar to the FgCelDH7C spectrum (Fig. 2a), which provides a possible spectroscopic feature for lower redox-potential AA7 enzymes.

Oxygen gating and activation at the re-side of the isoalloxazine ring in the FAD-domain are also important for the oxidase activity. The entry of $\mathrm{O}_{2}$ to the active site in flavo-enzymes is favoured through hydrophobic tunnels rather than the solventfilled substrate-binding pocket ${ }^{43}$. These tunnels converge to a cavity sterically gated by apolar residues ${ }^{33,44}$, where the $\mathrm{O}_{2}$ is positioned and activated for hydride transfer. Strikingly, we unveiled that the milieu of the FAD, particularly on the re-side, is considerably different in clade II as compared to canonical AA7s. An eye-catching substitution of a histidine, which is highly conserved in all other clades, to a serine, leucine or valine in clade II is observed (Fig. 6a, b, Supplementary Fig. 10, sequence patch 3). Mutation of a histidine at the equivalent position in the FADdependant aryl alcohol oxidase from Pleurotus eryngii was shown to reduce the rate of the oxidative half-reaction by an order of magnitude ${ }^{45}$. The histidine was proposed to contribute to the positioning and polarisation of $\mathrm{O}_{2}{ }^{45}$, thereby reducing its activation-free energy barrier. The substitution of this histidine in clade II enzymes is accompanied with other marked changes in the $\mathrm{O}_{2}$ binding cavity (Fig. 6a, b). These steric and chemical changes are likely to increase the energy barrier for hydride transfer to $\mathrm{O}_{2}{ }^{43,44}$, thereby severely reducing the oxidase activity. On the other hand, the uniquely open and positively charged active site of $\mathrm{FgCelDH7C}$, compared to structural homologues, may favour the accommodation of organic electron acceptors to promote the dehydrogenase oxidative half reaction. Taken together, these findings give insights into the structural elements associated with the distinctively high dehydrogenase/oxidase activity profile of $\mathrm{FgCelDH7C}$ and possibly AA7 homologues in clade II.

Clade I plant enzymes catalyse diverse reactions, e.g., in alkaloid synthesis ${ }^{32}$ and lignin building block synthesis demonstrated for the monolignol oxidase from Arabidopsis ${ }^{33}$. By contrast, the biological roles of oligosaccharide-specific oxidases have remained an enigma. Both canonical AA7 oligosaccharidespecific oxidases and clade II dehydrogenases are ubiquitous in phytopathogenic fungi and Oomycota plant parasites, responsible for some of the most devastating plant diseases in agriculture. Recently, the activity of oligogalacturonide oxidases (clade I) from Arabidopsis has been reported to reduce the elicitor activity of oligogalacturonides that trigger plant immune-response ${ }^{13}$. Interestingly, plants overexpressing the oligogalacturonide oxidase AtOGOX1 were more resistant to infection by the phytopathogen Botrytis cinerea, supporting a role in plant immunity ${ }^{13}$. Fungal LPMOs are another category of oxidoreductases that have been hypothesized to play a role in plant pathogenesis ${ }^{46}$, although this is yet to be demonstrated despite the abundance of LPMO genes in most fungal phytopathogens.

Interestingly, the co-secretion of fungal LPMOs and AA7 enzymes has been observed ${ }^{30,31}$, which inspired us to investigate the interplay between these two enzyme families. A balanced supply of ROS, e.g., $\mathrm{H}_{2} \mathrm{O}_{2}$ has been shown to be crucial for sustained LPMO activity, due to the affinity (low $\mu \mathrm{M}$ apparent $K_{\mathrm{M}}$ value $)^{22}$ of LPMOs to $\mathrm{H}_{2} \mathrm{O}_{2}$ and their high sensitivity to oxidative damage ${ }^{21,47}$. We have shown that $\mathrm{FgCelDH7C}$ potentiates LPMO-catalysed cellulose degradation in the absence of any exogenous reductant. The quenching of LPMO activity with either an alternative artificial acceptor for the AA7 (Supplementary Fig. 12) or an enzyme that scavenges $\mathrm{H}_{2} \mathrm{O}_{2}$ (Fig. 4a) is consistent with the proposed peroxygenase LPMO mechanism ${ }^{21}$. In addition, $\mathrm{O}_{2}{ }^{-}-$species that are generated by flavo-enzymes in the presence of $\mathrm{O}_{2}{ }^{48}$, may contribute to LPMO priming 
a

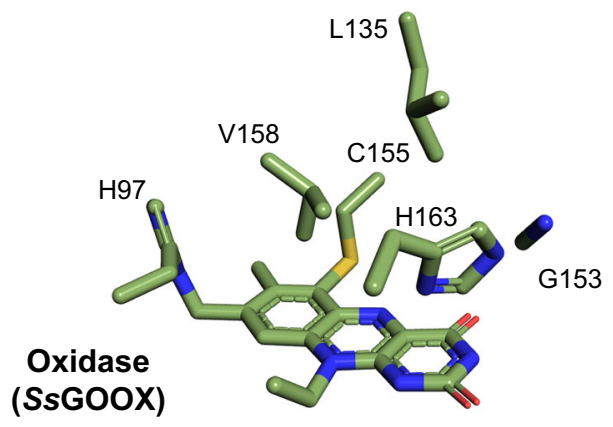

b

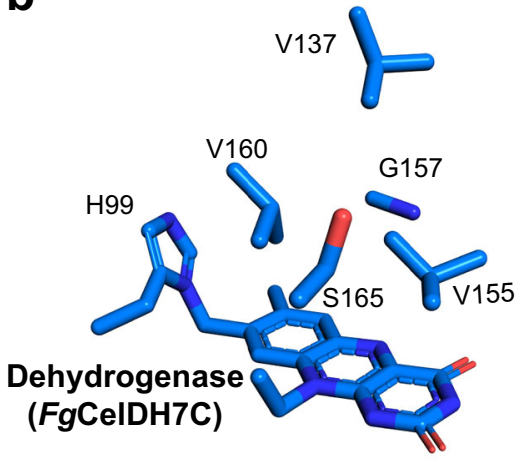

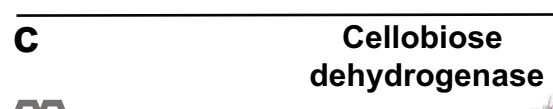

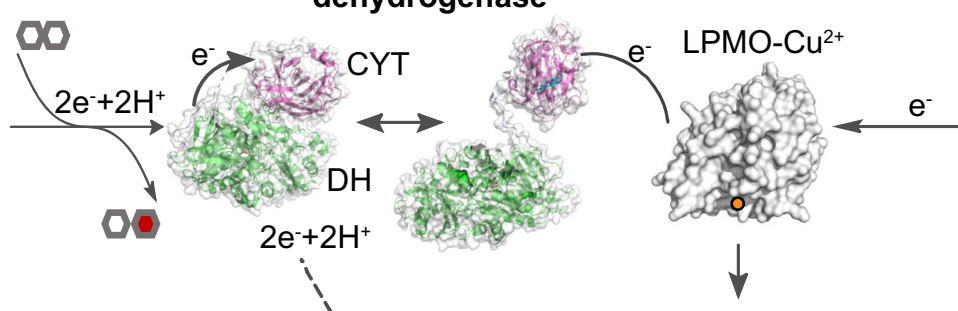

i

Cellulose
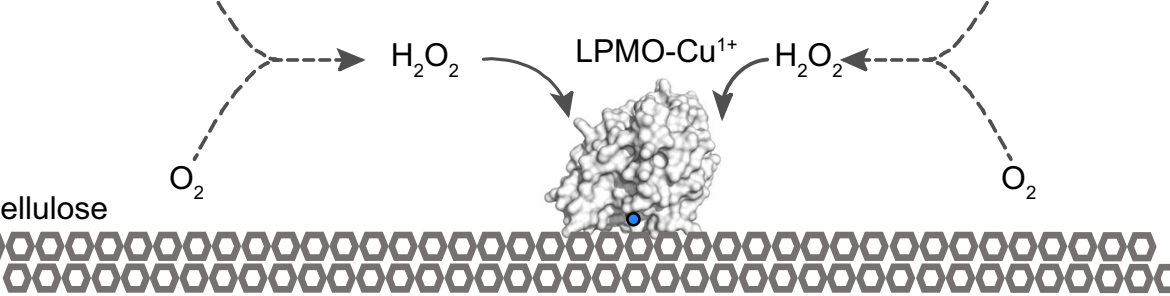

Cellobiose/cellotriose: $00 / 000$ Cellobiolactone/cellotrionolactone: 00 / 000

Fig. 6 Oxygen-binding cavity in AA7 oxidoreductases and a schematic model for AA7-LPMO interplay. a, b Putative oxygen-binding cavity in typical AA7 oxidases and dehydrogenases, respectively. The substitution of conserved histidine and glycine in oxidases to serine (or other small residues) and valine in dehydrogenases, respectively, is observed. c Schematic model of the interplay of cellobiose dehydrogenase (CDH) versus the AA7 cellooligosaccharide dehydrogenase with LPMOs during cellulose degradation. Both dehydrogenase classes oxidise cellooligosaccharides to the corresponding lactones. The electrons harvested from this oxidation are stored in the FAD-cofactor and subsequently delivered directly to the LPMO in the case of AA7. The transfer of priming electrons in $\mathrm{CDH}$ proceeds first from the dehydrogenase domain to the cytochrome $b$ haem domain in (closed form). A subsequent large conformational change to the open form is required to expose the haem domain to the LPMO active site for electron transfer. The dotted lines signify the low oxidase side-activity from both classes of dehydrogenases, which generates the $\mathrm{H}_{2} \mathrm{O}_{2}$ preferred co-substrate to fuel cellulose oxidative degradation by LPMOs. Low levels of $\mathrm{H}_{2} \mathrm{O}_{2}$ are also generated at the active site of free primed LPMOs, but this is left out from the figure for clarity.

consistent with previous findings ${ }^{21,38}$. To evaluate the specificity of the observed AA7-mediated activation of LPMOs, MoChi7A that possesses a low oxidase activity on cellooligosaccharides was used instead of $\mathrm{FgCelDH7C}$ at equimolar concentrations in a similar assay. Under these conditions, the release of oligomers from cellulose was not observed to the same extent, indicating that the priming and/or the balanced fuelling of LPMO activity is more readily achieved with the AA7 dehydrogenase (Supplementary Fig. 15). Remarkably, the performance of FgCelDH7C was comparable with $\mathrm{CDH}$ that has been shown to mediate direct transfer of priming electrons from cellooligosaccharide oxidation to LPMOs via the cytochrome $b$ domain ${ }^{49}$. Our EPR experiments were consistent with direct electron transfer to the LPMO when the FAD-cofactor in FgCelDH7C was reduced either with dithionite or cellotriose in the absence of $\mathrm{O}_{2}$ (Fig. $5 \mathrm{c}, \mathrm{d}$ ). This electron transfer and the interaction with the LPMO is likely to occur at the substrate-binding face (si-side) of the FAD, which is consistent with the rather open active site topology of FgCelDH7C (Supplementary Fig. 9). By comparison, the electron transfer from the larger (about $800 \mathrm{aa}$ ) and bi-modular $\mathrm{CDH}$ to the LPMO requires inter-domain electron transfer from the dehydrogenase to the cytochrome domain and a subsequent large domain movement to the open $\mathrm{CDH}$ form ${ }^{49,50}$. The deletion of the cytochrome domain impairs the priming of the LPMO, but the LPMO fuelling remains efficient ${ }^{29}$, likely via the oxidase sideactivity of $\mathrm{CDH}$. Similarly, the deletion of the cytochrome domain in the pyrroloquinoline-quinone-dependent pyranose dehydrogenase impaired the fuelling of LPMO activity ${ }^{24}$. In summary, the one-module AA7 dehydrogenase confers direct priming of LPMOs in contrast to other known LPMO redox partners that require either small organic molecules or an additional cytochrome haem domain to mediate LPMO priming.

The expansion of the substrate range of fungal oligosaccharideoxidising flavo-enzymes belonging to the AA7 family and the potentiation of LPMO activity, highlight the functional diversity within AA7. These findings set the stage for harnessing other AA7 dehydrogenases to fine-tune the activity of LPMOs that lack known redox partners. The demonstration of a functional and 
efficient AA7-LPMO system offers attractive avenues for the development of commercial enzyme blends targeting recalcitrant biomass.

\begin{abstract}
Methods
Bioinformatics and phylogenetic analysis. The sequences of 2200 putative AA7 sequences were retrieved by a BlastP search against the non-redundant protein database interfaced the NCBI server (https://blast.ncbi.nlm.nih.gov/Blast. cgi) using the $F g C h i t O$ sequence (Genbank accession: XP_011325372) as a query and default search settings. The retrieved sequences were filtered by excluding noneukaryotic proteins and those displaying $<25 \%$ amino acid (aa) identity and $<90 \%$ coverage to the query. Two additional AA7-like sequences, which were differentially upregulated in the secretome of Aspergillus nidulans grown on starch ${ }^{31}$, and a longer sequence possessing an $\mathrm{N}$-terminal extension of about 250 amino acid residues (aa) (MoChi7A) were also included in the sequence inventory. Only sequences comprising 470-570 aa $(n=1927)$ were aligned using MAFFT ${ }^{51}$ with default parameters, and curated using Gblock $0.91 \mathrm{~b}^{52}$. The alignment was used to construct a phylogenetic tree using NGphylogeny ${ }^{53,54}$ and rendered by iTOL ${ }^{54}$. The visualization of amino acid conservation was made using WebLogo ${ }^{55}$.
\end{abstract}

Production of selected AA7 enzymes. Based on the phylogenetic analysis above, five selected AA7 gene fragments encoding mature peptides without signal peptides (Supplementary Table 1) were codon optimised for P. pastoris and purchased from GENEWIZ (NJ, USA). The gene fragments were cloned within the XbaI and XhoI restriction sites of the pPICZaA vector (Invitrogen, Carlsbad, CA, USA) in frame with the Saccharomyces cerevisiae a-mating factor secretion signal and fused to a C-terminal (His) ${ }_{6}$ tag. The synthetic geneharbouring plasmids were propagated in Escherichia coli DH5a, linearised with PmeI and thereafter transformed into competent $P$. pastoris X33 by electroporation following the protocols from the Easy Select Expression System (Invitrogen). Six transformants per construct were screened for production in deep well plates for three days with $\mathrm{MeOH}$ addition 3\% (v/v) every $24 \mathrm{~h}$. The best-secreting transformants based on SDS-PAGE gel electrophoresis, from the constructs of the selected enzymes (Supplementary Table 1) were chosen for larger-scale production. These clones were grown in shake flasks containing $2 \mathrm{~L}$ of BMGY (Pichia expression manual, Invitrogen) containing $1 \mathrm{~mL} \mathrm{~L}^{-1}$ of Pichia PTM4 trace element solution $\left(2 \mathrm{~g} \mathrm{~L}^{-1} \mathrm{CuSO}_{4} \cdot 5 \mathrm{H}_{2} \mathrm{O}, 3 \mathrm{~g} \mathrm{~L}^{-1}, \mathrm{MnSO} 4 . \mathrm{H}_{2} \mathrm{O}\right.$, $0.2 \mathrm{~g} \mathrm{~L}^{-1} \mathrm{Na}_{2} \mathrm{MoO}_{4} \cdot 2 \mathrm{H}_{2} \mathrm{O}, 0.02 \mathrm{~g} \mathrm{~L}^{-1} \mathrm{H}_{3} \mathrm{BO}_{3}, 0.5 \mathrm{~g} \mathrm{~L}^{-1} \mathrm{CaSO}_{4} \cdot 2 \mathrm{H}_{2} \mathrm{O}, 0.5 \mathrm{~g} \mathrm{~L}^{-1}$ $\mathrm{CoCl}_{2}, 12.5 \mathrm{~g} \mathrm{~L}^{-1} \mathrm{ZnSO}_{4} .7 \mathrm{H}_{2} \mathrm{O}, 22 \mathrm{~g} \mathrm{~L}^{-1}, \mathrm{FeSO}_{4} .7 \mathrm{H} 2 \mathrm{O}, \mathrm{H}_{2} \mathrm{SO}_{4} 1 \mathrm{~mL} \mathrm{~L}^{-1}$ ) and biotin $0.2 \mathrm{~g} \mathrm{~L}^{-1}$ to an $O D_{600}$ of $2-6$, for $16 \mathrm{~h}$ at $30^{\circ} \mathrm{C}$ using an orbital shaker $(200 \mathrm{rpm})$. The cells from each construct were harvested by centrifugation $\left(4000 \times g, 10 \mathrm{~min}, 4^{\circ} \mathrm{C}\right)$ and expression was induced by re-suspending the cells into $400 \mathrm{~mL}$ of BMMY medium at $20^{\circ} \mathrm{C}$ and the culture was continued for 3 days with methanol supplementation to $3 \%(\mathrm{v} / \mathrm{v})$ every $24 \mathrm{~h}$. The cells were harvested by centrifugation $\left(5,000 \times g, 10 \mathrm{~min}, 4^{\circ} \mathrm{C}\right)$ and the $\mathrm{pH}$ of the culture supernatants was adjusted to 7.8 using $\mathrm{NaOH}(2 \mathrm{M})$, followed by sterile filtration using $0.22 \mu \mathrm{m}$ filters (Millipore, Burlington, MA, USA). The filtered supernatants were loaded onto $5 \mathrm{~mL}$ His Trap HP columns (GE Healthcare, Uppsala, Sweden) connected to an Äkta purifier 100 (GE Healthcare) at

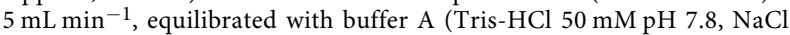
$150 \mathrm{mM}$, imidazole $10 \mathrm{mM}$ ). Non-bound proteins were washed by 10 column volumes (CVs). The bound proteins were eluted with a $34 \%$ buffer B (Tris- $\mathrm{HCl}$ $50 \mathrm{mM} \mathrm{pH} 7.8, \mathrm{NaCl} 150 \mathrm{mM}$, imidazole $500 \mathrm{mM}$ ) gradient in $10 \mathrm{CVs}$. Fractions containing recombinant enzymes were pooled, concentrated and buffer exchanged against $50 \mathrm{mM} \mathrm{NaOAc}$, pH 5.2 using a $10 \mathrm{kDa}$ Vivaspin ultrafiltration unit (Sartorius, Göttingen, Germany). Protein purity was assessed using SDS-PAGE analysis and enzyme concentration was determined by measuring $A_{280}$ using a Nanodrop ND-2000 (Thermo Fisher Scientific, Waltham, MA, USA) and the theoretically calculated molar extinction coefficients (Table S1) using the Protparam tool on the EXPASY server (web.expasy.org/ protparam/).

\section{Activity assays and kinetic analyses}

Initial screening assay. The oxidase activity assay of the expressed MoChi7A, $\mathrm{FgChi7B}, \mathrm{FgCelDH7C}$ and $\mathrm{AnAA7A}$ was conducted by coupling $\mathrm{H}_{2} \mathrm{O}_{2}$ production to HRP mediated oxidation of 4-aminoantipyrine (AAP) and 3,5-dichloro-2hydroxybenzensulfonic acid (DCHBS) (both from Sigma Aldrich, St. Louis, MI, USA) in 96-well microtiter plates. Reactions ( $200 \mu \mathrm{L}, 0.1 \mathrm{mM}$ AAP, $1 \mathrm{mM}$ DCHBS, $8 \mathrm{U} \mathrm{mL}^{-1} \mathrm{HRP}$ (Sigma Aldrich) in $50 \mathrm{mM} \mathrm{NaOAc}$ buffer, $\mathrm{pH}$ 5.2) were monitored by measurements of $A_{505 \mathrm{~nm}}$ at $35^{\circ} \mathrm{C}$ for 20 min with $30 \mathrm{~s}$ intervals. The assay was performed in triplicates using $0.1 \mu \mathrm{M} \mathrm{AA7}$ against 30 carbohydrates, two sugar alcohols (all $2 \mathrm{mM}$ except $1 \mathrm{mM}$ for LNB/GNB) and 6 aromatic alcohols ( $1 \mathrm{mM}$ ) (Supplementary Table 2) by the addition of $100 \mu \mathrm{L}$ substrate into pre-temperated reaction mixtures in microtiter plates. The substrate screening of $P b C h i 7 \mathrm{~A}$ was conducted using a modified reaction set up $(250 \mu \mathrm{L}, 0.1 \mathrm{mM}$ AAP, $1 \mathrm{mM}$ phenol, 8 $\mathrm{U} \mathrm{mL}^{-1} \mathrm{HRP}$ (Sigma Aldrich) in $50 \mathrm{mM}$ Tris-HCl pH 8.0) incubated in triplicates at $37^{\circ} \mathrm{C}$ for $20 \mathrm{~min}$ and measured sequentially at $A_{505} \mathrm{~nm}$ with $30 \mathrm{~s}$ intervals.
Oxidation kinetics of AA7. The steady-state apparent kinetic parameters of MoChi7 A and FgChi7B $(0.65 \mu \mathrm{M})$ were determined in an analogous assay, where the reaction mixtures $(200 \mu \mathrm{L})$ were monitored for 3 min with $20 \mathrm{~s}$ measurements intervals. The initial rates for the oxidation of $\mathrm{N}$-acetylglucosamine (GlcNAc) and $\mathrm{N}$-acetylgalactosamine (both in $0.09-12.5 \mathrm{mM}$ ) (GalNAc) (only for MoChi7A), chitobiose $(0.0-0.5 \mathrm{mM})$ and chitotriose $(0.03-0.6 \mathrm{mM})$ were determined in triplicates. The kinetic parameters of $\mathrm{PbChi7A}$ were determined using the same HRP assay on 12 substrates, including mono- $(1-1500 \mathrm{mM})$, di- $(1-250 \mathrm{mM})$, trisaccharides $(0.05-25 \mathrm{mM})$ (Supplementary Table 2). The kinetic parameters were determined by fitting the Michaelis-Menten equation in Origin, versions 9.55 and 18 (Northampton, Massachusetts, USA) to the initial rates, determined from the slopes of the linear reaction phases (Table 1). Due to the low oxidase activity of $\mathrm{FgCelDH7C}$, the activity assay was performed at higher enzyme concentrations $(150$ and $500 \mu \mathrm{M})$ in duplicates each and the absorbance was measured for one hour with 1 min intervals to determine the normalised reaction rate $\left(V_{\mathrm{o}} / \mathrm{E}\right)$.

Dehydrogenase assay of AA7 activity. The dehydrogenase activity of the enzymes was also assayed using the redox mediator 2,6-dichlorophenolindophenol (DCIP) $\left(\varepsilon_{520}=6.8 \mathrm{mM}^{-1} \mathrm{~cm}^{-1}\right.$ ) as a terminal electron acceptor (as opposed to $\mathrm{O}_{2}$ for the oxidase activity). Reactions ( $200 \mu \mathrm{L}, 0.1 \mathrm{mM}$ DCIP, $5 \mathrm{mM}$ carbohydrate or $1 \mathrm{mM}$ alcohol substrates, $0.65 \mu \mathrm{M}$ enzyme in $50 \mathrm{mM}$ HEPES, $\mathrm{pH}$ 7.0) were initiated by adding $100 \mu \mathrm{L}$ carbohydrates or alcohol substrates to $100 \mu \mathrm{L}$ pre-temperated mixtures containing all other components. The decrease in $A_{530}$ was measured continuously for $20 \mathrm{~min}$ with $30 \mathrm{~s}$ intervals at $35^{\circ} \mathrm{C}$.

Temperature and $\mathrm{pH}$ stability/optimum measurements. The $\mathrm{pH}$-dependence of the activity of $\mathrm{MoChi7A}, \mathrm{FgChi7B}$ and $\mathrm{FgCelDH7C} \mathrm{(100} \mathrm{nM} \mathrm{for} \mathrm{all)} \mathrm{towards} 2 \mathrm{mM}$ of GlcNAc, chitobiose and cellobiose, respectively, was analysed in triplicates using the dehydrogenase assay as above (DCIP as a terminal electron acceptor). The assay was carried out at eight $\mathrm{pH}$ values in the range 5.1-9.0 by exchanging the buffer in the screening assay to the universal Britton-Robinson buffer (acetic acid, phosphoric acid, and boric acid, $50 \mathrm{mM}$ of each). The dehydrogenase assay was used to allow for comparison of all three enzymes in the same assay and the initial rates of the oxidation at each $\mathrm{pH}$ value we determined from the linear parts of the progress curves as above. Differential scanning calorimetry was employed to examine the conformational stability of $\mathrm{FgCelDH7C}\left(1 \mathrm{mg} \mathrm{mL}^{-1}\right)$ at $\mathrm{pH}$ values of 5.2 and 7.2 in $50 \mathrm{mM}$ (in acetate and phosphate buffers, respectively) in the temperature range of $15-90^{\circ} \mathrm{C}$ at a scanning rate of $1^{\circ} \mathrm{C} \mathrm{min}-1$ using a NanoDSC instrument (TA instruments, New Castle, DE, USA).

The temperature and $\mathrm{pH}$ optima of $P b C h i 7 \mathrm{~A}(15 \mathrm{nM})$ were determined using the HRP assay described above against chitobiose $(2 \mathrm{mM})$. For determination of the $\mathrm{pH}$ optimum, a Britton Robinson buffer (100 mM each) was adjusted to $\mathrm{pH} 4-11$ and the oxidation activity was measured after $1 \mathrm{~h}$ incubation at $4{ }^{\circ} \mathrm{C}$. The temperature stability of PbChi7A $(15 \mathrm{nM})$ was measured by oxidation of $2 \mathrm{mM}$ chitobiose within $1 \mathrm{~h}$ at different temperatures $\left(25^{\circ} \mathrm{C}-55^{\circ} \mathrm{C}\right)$.

NMR Spectroscopy of FgCelDH7C, FgChi7B and MoChi7A. Initially, the analysis of post oxidation reactions was conducted on a reaction mixture containing $\mathrm{FgChi7B}$ or $\mathrm{MoChi7A}(1 \mu \mathrm{M}$ of each), $25 \mathrm{mM}$ GalNAc or GlcNAc as substrates, buffered with $50 \mathrm{mM} \mathrm{NaOAc}$ buffer pH $5.2\left(500 \mu \mathrm{L}, 25^{\circ} \mathrm{C}, 30 \mathrm{~min}\right)$. The reaction mixtures were ice-cooled and immediately analysed at $15^{\circ} \mathrm{C}$. Spectra were collected using an $800 \mathrm{MHz}$ Bruker Avance II spectrometer equipped with an Oxford $18.7 \mathrm{~T}$ magnet and a TCI cryoprobe by transferring $500 \mu \mathrm{L}$ of the post-reaction mixtures to NMR sample tubes $(5 \mathrm{~mm})$ and performing ${ }^{1} \mathrm{H},{ }^{1} \mathrm{H}-{ }^{1} \mathrm{H}$ TOCSY, ${ }^{1} \mathrm{H}-{ }^{1} \mathrm{H}$ COSY, ${ }^{1} \mathrm{H}_{-}{ }^{13} \mathrm{C}$ HMBC NMR and multiplicity-edited ${ }^{1} \mathrm{H}_{-}{ }^{13} \mathrm{C}$ HSQC NMR spectroscopy analyses. ${ }^{1} \mathrm{H}-{ }^{1} \mathrm{H}$ TOCSY spectra were acquired as data matrices of $1024 \times 256$ complex data points sampling 128 and $64 \mathrm{~ms}$ in the direct and indirect dimensions, respectively. ${ }^{1} \mathrm{H}-{ }^{1} \mathrm{H}$ COSY spectra were acquired as data matrices of $2048 \times 128$ complex data points sampling 320 and $20 \mathrm{~ms}$ in the direct and indirect dimensions, respectively, while ${ }^{1} \mathrm{H}-{ }^{13} \mathrm{C}$ HMBC NMR spectra were acquired as data matrices of $2048 \times 256$ complex data points sampling 212 and $5.8 \mathrm{~ms}$ in the direct and indirect dimensions, respectively. Multiplicity-edited ${ }^{1} \mathrm{H}_{-}{ }^{13} \mathrm{C}$ HSQC spectra were collected by sampling the NMR spectra for 106 and $15.9 \mathrm{~ms}$ in the direct $\left({ }^{1} \mathrm{H}\right)$ and indirect $\left({ }^{13} \mathrm{C}\right)$ dimensions, respectively. Once intermediate and product identification was established (Supplementary Fig. 3), a series of ${ }^{1} \mathrm{H}^{13} \mathrm{C}$ HSQC spectra was acquired at $15^{\circ} \mathrm{C}$ to follow the conversion of GlcNAc and GalNAc by MoChi7A $(1 \mu \mathrm{M})$ in $50 \mathrm{mM} \mathrm{NaOAc}$ buffer pH 5.2 after 5, 15, 60 and $180 \mathrm{~min}$ experiment time. The ${ }^{1} \mathrm{H}-$ ${ }^{13} \mathrm{C}$ HSQC spectra were collected by sampling the NMR spectra for 159 and $8.3 \mathrm{~ms}$ in the direct $\left({ }^{1} \mathrm{H}\right)$ and indirect $\left({ }^{13} \mathrm{C}\right)$ dimensions, respectively, with experiments of 19 min duration to yield the time course shown in Supplementary Fig. 4 upon integration of the $\mathrm{C} 2 \mathrm{H} 2 \mathrm{NMR}$ cross peaks.

For cellobiose, a time series of ${ }^{1} \mathrm{H}-{ }^{13} \mathrm{C}$ HSQC spectra for the enzymatic conversion was acquired using $\mathrm{FgCelDH7C}(0.56 \mu \mathrm{M})$ to yield assignments of cellobionolactone and of cellobionic acid signals as displayed in Supplementary Figure 5. Time-resolved ${ }^{1} \mathrm{H}$ NMR spectra sampling 16384 complex data points of the FID for $1.27 \mathrm{~s}$ were subsequently used to track the reaction kinetics for cellobiose $(2 \mathrm{mM})$ conversion by $\mathrm{FgCelDH7C}(0.56 \mu \mathrm{M})$ at $25^{\circ} \mathrm{C}$ in the presence or in the absence of $1.3 \mathrm{mM}$ DCIP as an electron acceptor using the same equipment as above. The ${ }^{1} \mathrm{H}$ singlet signal for DCIP as well as signals for $\mathrm{H} 5$ of cellobionolactone and $\mathrm{H} 2$ of cellobionic acid were integrated alongside the $\mathrm{H} 2$ and 
H3 signals for the reducing end of cellobiose. Signal areas were normalized to the number of contributing hydrogen atoms. The same buffer, enzyme concentration and same NMR setup were subsequently used to track the dependence of cellotetraose oxidation $(6 \mathrm{mM}$ cellotetraose) by $\mathrm{FgCelDH7C}$ in the presence of $0.025,0.05,0.1,0.2,0.5$ or $1 \mathrm{mM}$ DCIP. All NMR spectra were acquired, processed and integrated in Topspin 3.5 pl6 with ample zero filling in all dimensions.

\section{Crystallization and structure Determination of FgCelDH7C and FgChi7B}

Crystallization screens PACT ++ (Jena Bioscience, Jena, Germany), JCSG+ and Morpheus screens (Molecular Dimensions, Sheffield, UK) and optimisation plates of FgCelDH7C ( $9.0 \mathrm{mg} \mathrm{mL}^{-1}$, deglycosylated with EndoH, New England Biolab,

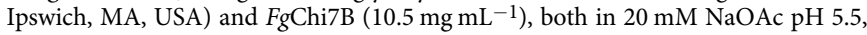
were performed with a Crystal Gryphon liquid handling robot (Art Robbins Instruments, Sunnyvale, USA). Drops containing equal volumes of protein and reservoir solutions were mixed. Crystals of $\mathrm{FgCelDH7C}$ appeared in the F7 condition (NaSCN, 20\% (w/v) PEG 3350), which was optimised by varying PEG content $(12.5-25 \% \mathrm{w} / \mathrm{v})$. The best crystals formed within 4 days at $20^{\circ} \mathrm{C}$, using 150 $\mathrm{nL}$ of reservoir and protein solutions (1:1 ratio) in $50 \mu \mathrm{l}$ reservoir solution. These crystals were cryo-protected with PEG400 in reservoir solution and cryo-cooled in a nylon loop. Crystals of $\mathrm{FgChi7B}$ were initially observed in PACT ++ condition E10 (PEG 3350, 22\% w/v, $200 \mathrm{mM}$ sodium potassium phosphate using a 1:1 protein:reservoir ratio. Crystals were optimised by micro-seeding using the above condition at 22 and 24\% PEG (w/v). Drops contained 1:1:0.2 of protein:reservoir: seed stock (100 fold diluted seed stock) ratio, respectively. The crystals were cryocooled using a mixture of reservoir and PEG400 (0.7:0.3 ratio) added to the drop prior freezing in liquid nitrogen. Diffraction data for $\mathrm{FgCelDH7C}$ and $\mathrm{FgChi7B}$ crystals were collected in BioMax beamline at MaxIV (Lund, Sweden) using MXCuBE v3 and id30A3 (MASSIF-3) beamline at ESRF (Grenoble, France) using to $1.64 \AA$ and $2.38 \AA$ resolution, respectively. Both structures were solved using molecular replacement in Phaser ${ }^{56}$ with model coordinates of $\left.T t \mathrm{XylO}\right]$ and initial automated model building and refinement using PHENIX.autobuild ${ }^{57}$ and PHENIX.refine ${ }^{58}$, respectively. Manual model rebuilding and map inspection were performed in $\operatorname{Coot}^{59}$ and analysed using MolProbity ${ }^{60}$. The final validated structural models of $\mathrm{FgCelDH} 7 \mathrm{C}$ and $\mathrm{FgChi7B}$ were deposited to the Protein Data Bank under the entry codes 6YJI and 6YJO], respectively (Data collection and refinement statistics are in Supplementary Table 4).

\section{Cellulose degradation assays based on the interplay between LPMOs and}

AA7 enzymes. The assays were performed using suspensions of either $1 \% \mathrm{PASC}^{61}$ and/or 0.5\% Avicel (Honeywell Fluka, Morris Plains, NJ, U.S.A) (both w/v) in 250 or $500 \mu \mathrm{L}$ containing $4.4 \mu \mathrm{M}$ of PaLPMO9E ${ }^{20}$, PaLPMO$^{20} \mathrm{H}^{20}$ or $L s \mathrm{AA} 9 \mathrm{~A}^{35}$ as well as $0.4 \mu \mathrm{M} \mathrm{FgCelDH7C}(0.2 \mu \mathrm{M}$ in case of LsAA9A) in $50 \mathrm{mM} \mathrm{NaOAc}$, pH 5.2 in the presence of cellotetraose (DP4, $0.8 \mathrm{mM}$ ), unless stated otherwise. For the comparison of $\mathrm{FgCelDH7C}$ and $\mathrm{MoChi7A}, 0.2 \mu \mathrm{M}$ of each was used. Controls were also performed in absence of individual reaction components or in the presence of $0.6 \mathrm{mM}$ DCIP. Moreover, the AA7-mediated LPMO activity was compared to reactions in the presence of ascorbate $(1 \mathrm{mM})$, L-Cys $(1 \mathrm{mM})$, reactions containing horseradish peroxidase (HRP, $0.2-38 \mathrm{nM}$ in the presence of $0.1 \mathrm{mM}$ AAP, $1 \mathrm{mM}$ DCHBS), superoxide dismutase (SOD, recombinantly expressed in E. coli, $0.07-0.5$ $\mu \mathrm{M}$, Sigma Aldrich) in $100 \mu \mathrm{M}$ phosphate, $\mathrm{pH}$ 7.0, or CDH from Podospora anserina $(\mathrm{PaCDH}, 1.2 \mu \mathrm{M})^{34}$.

The reactions were performed in $2 \mathrm{~mL}$ Eppendorf tubes with $850 \mathrm{rpm}$ stirring in thermomixer (Eppendorf, Hamburg, Germany) at $35^{\circ} \mathrm{C}$ for $2-24 \mathrm{~h}$ and thereafter quenched by boiling $\left(100^{\circ} \mathrm{C}, 10 \mathrm{~min}\right)$ and/or fourfold dilution in $\mathrm{NaOH}$ to $0.1 \mathrm{M}$ followed by centrifugation $\left(15,000 \times g, 15 \mathrm{~min}, 4^{\circ} \mathrm{C}\right)$ to separate the released saccharides in the supernatants from the insoluble fraction prior to analysis. The solubilised oxidised and non-oxidised oligosaccharides, released by the enzymatic treatments, were analysed by high-performance anion-exchange chromatography coupled with pulsed amperometric detection (HPAEC-PAD) (ThermoFischer Scientific) using a CarboPac ${ }^{\text {mix }}$ PA1 (ThermoFischer Scientific) and a CarboPac ${ }^{\text {Tx }}$ PA1 guard column $(2 \times 50 \mathrm{~mm})$ at $0.25 \mathrm{~mL} \mathrm{~min}^{-1}$ and an eluent system as described by Westereng et al. ${ }^{62}$. The Chromeleon 6.80 SR10 software was used to interface the instrument and analyse the chromatograms. Non-oxidised oligosaccharides were used as standards (Megazyme, Wicklow, Ireland), whereas the corresponding C1-oxidised standards (from DP2-DP6) were provided from native cello-oligosaccharides using $\mathrm{PaCDHB}$ treatment ${ }^{34}$.

EPR spectroscopy. EPR experiments were performed on a Bruker ELEXSYS E500 $\mathrm{X}$-band spectrometer equipped with an ER4102ST standard rectangular Bruker EPR cavity fitted to an Oxford Instruments ESR 900 helium flow cryostat. All EPR studies were carried out at $50 \mathrm{~K}$ with $4 \mathrm{~mW}$ microwave power at $9.479 \mathrm{GHz}$ and 3 $\mathrm{mT}$ modulation amplitude at $100 \mathrm{kHz}$. The presented spectra were averaged from the accumulation of 4 scans to improve the signal-to-noise ratio. The EPR samples were prepared under anaerobic conditions in glove box (Jacomex) and frozen prior to the EPR analysis. In a first experiment, two $160 \mu \mathrm{L}$ protein samples were prepared in $50 \mathrm{mM} \mathrm{NaOAc}$ pH 5.2 and incubated at $25^{\circ} \mathrm{C}$ for $5 \mathrm{~min}$ : (i) control mixtures of PaLPMO9H (100 $\mu \mathrm{M}$ ) and cellotriose (DP3, $1 \mathrm{mM}$ ), (ii) same as (i), but supplemented with $\mathrm{FgCelDH7C}(10 \mu \mathrm{M})$.
In the second set of experiments, PaLPMO9H $(20 \mu \mathrm{M})$ was prepared in the same buffer in the absence or presence of $40 \mu \mathrm{M}$ dithionite and incubated at $25^{\circ} \mathrm{C}$ for $15 \mathrm{~min}$ to achieve the full reduction of the catalytic $\mathrm{Cu}(\mathrm{II})$ center of $\mathrm{PaLPMO} 9 \mathrm{H}$ into $\mathrm{Cu}(\mathrm{I})$ by dithionite. Next, the following solutions were prepared and incubated anaerobically for $5 \mathrm{~min}$ at $25^{\circ} \mathrm{C}$ : (i) $\mathrm{FgCelDH} 7 \mathrm{C}(20 \mu \mathrm{M})$ with substoichiometric amount of sodium dithionite $(16 \mu \mathrm{M})$ to reduce $80 \%$ of the flavoenzyme, while avoiding excess dithionite in the solution, (ii) PaLPMO9H $(20 \mu \mathrm{M})$ without additives in the same buffer. Equal volumes of the two solutions were then pooled into a $160 \mu \mathrm{L}$ EPR sample and incubated for few minutes prior freezing and EPR analysis.

Reporting summary. Further information on research design is available in the Nature Research Reporting Summary linked to this article.

\section{Data availability}

The atomic coordinates of $\mathrm{FgCelDH7C}$ and $\mathrm{FgChi7B}$ have been deposited in the Protein Data Bank (https://www.rcsb.org) under the PDB accessions 6YJI and 6YJO, respectively (see also Supplementary Table 4). The GenPept accession IDs of the enzymes characterised in the study are given in Supplementary Table 1. All the data are available from the corresponding authors upon request. Source data are provided with this paper

Received: 26 October 2020; Accepted: 9 March 2021; Published online: 09 April 2021

\section{References}

1. Eriksson, K. E., Pettersson, B. \& Westermark, U. Oxidation-important enzyme reaction in fungal degradation of cellulose. FEBS Lett. 49, 282-285 (1974).

2. Lo Leggio, L. et al. Structure and boosting activity of a starch-degrading lytic polysaccharide monooxygenase. Nat. Commun. 6, 5961 (2015).

3. Vu, V. V., Beeson, W. T., Span, E. A., Farquhar, E. R., and Marletta, M. A. A family of starch-active polysaccharide monooxygenases. Proceedings of the National Academy of Sciences 111, 13822-13827 https://doi.org/10.1073/ pnas.1408090111 (2014)

4. Haddad Momeni, M. et al. Loss of AA13 LPMOs impairs degradation of resistant starch and reduces the growth of Aspergillus nidulans. Biotechnol. Biofuels 13, 135 (2020).

5. Vaaje-Kolstad, G. et al. An oxidative enzyme boosting the enzymatic conversion of recalcitrant polysaccharides. Science 330, 219-222 (2010).

6. Quinlan, R. J. et al. Insights into the oxidative degradation of cellulose by a copper metalloenzyme that exploits biomass components. Proc. Natl Acad. Sci. USA 108, 15079-15084 (2011).

7. Beeson, W. T., Phillips, C. M., Cate, J. H. D. \& Marletta, M. A. Oxidative cleavage of cellulose by fungal copper-dependent polysaccharide monooxygenases. J. Am. Chem. Soc. 134, 890-892 (2012).

8. Filiatrault-Chastel, C. et al. AA16, a new lytic polysaccharide monooxygenase family identified in fungal secretomes. Biotechnol. Biofuels 12, 55 (2019).

9. Nekiunaite, L. et al. FgLPMO9A from Fusarium graminearum cleaves xyloglucan independently of the backbone substitution pattern. FEBS Lett. 590, 3346-3356 (2016).

10. Couturier, M. et al. Lytic xylan oxidases from wood-decay fungi unlock biomass degradation. Nat. Chem. Biol. 14, 306-310 (2018).

11. Bissaro, B., Várnai, A., Røhr, Å. K. \& Eijsink, V. G. H. Oxidoreductases and reactive oxygen species in conversion of lignocellulosic biomass. Microbiol. Mol. Biol. Rev. 82, e00029-00018 (2018).

12. Lombard, V., Ramulu, H. G., Drula, E., Coutinho, P. M. \& Henrissat, B. The carbohydrate-active enzymes database (CAZy) in 2013. Nucleic Acids Res. 42, D490-D495 (2014).

13. Benedetti, M. et al. Four Arabidopsis berberine bridge enzyme-like proteins are specific oxidases that inactivate the elicitor-active oligogalacturonides. Plant J. 94, 260-273 (2018).

14. Locci, F. et al. An Arabidopsis berberine bridge enzyme-like protein specifically oxidizes cellulose oligomers and plays a role in immunity. Plant $J$. 98, 540-554 (2019).

15. Lee, M. H. et al. Structural characterization of glucooligosaccharide oxidase from Acremonium strictum. Appl Environ. Microbiol. 71, 8881-8887 (2005)

16. $\mathrm{Xu}, \mathrm{F}$. et al. A novel carbohydrate:acceptor oxidoreductase from Microdochium nivale. Eur. J. Biochem. 268, 1136-1142 (2001).

17. Ferrari, A. R. et al. Discovery of a xylooligosaccharide oxidase from Myceliophthora thermophila C1. J. Biol. Chem. 291, 23709-23718 (2016).

18. Heuts, D. P., Janssen, D. B. \& Fraaije, M. W. Changing the substrate specificity of a chitooligosaccharide oxidase from Fusarium graminearum by model-inspired site-directed mutagenesis. FEBS Lett. 581, 4905-4909 (2007). 
19. Savino, S., Jensen, S., Terwisscha van Scheltinga, A. \& Fraaije, M. W. Analysis of the structure and substrate scope of chitooligosaccharide oxidase reveals high affinity for C2-modified glucosamines. FEBS Lett. 594, 2819-2828 (2020).

20. Leferink, N. G., Heuts, D. P., Fraaije, M. W. \& van Berkel, W. J. The growing VAO flavoprotein family. Arch. Biochem. Biophys. 474, 292-301 (2008).

21. Bissaro, B. et al. Oxidative cleavage of polysaccharides by monocopper enzymes depends on $\mathrm{H}_{2} \mathrm{O}_{2}$. Nat. Chem. Biol. 13, 1123-1128 (2017).

22. Kuusk, $\mathrm{S}$. et al. Kinetics of $\mathrm{H}_{2} \mathrm{O}_{2}$-driven degradation of chitin by a bacterial lytic polysaccharide monooxygenase. J. Biol. Chem. 293, 523-531 (2018).

23. Jones, S. M., Transue, W. J., Meier, K. K., Kelemen, B. \& Solomon, E. I. Kinetic analysis of amino acid radicals formed in $\mathrm{H}_{2} \mathrm{O}_{2}$-driven $\mathrm{Cu}^{\mathrm{I}} \mathrm{LPMO}$ reoxidation implicates dominant homolytic reactivity. Proc. Natl Acad. Sci. 201922499 (2020).

24. Varnai, A., Umezawa, K., Yoshida, M. \& Eijsink, V. G. H. The pyrroloquinoline-quinone-dependent pyranose dehydrogenase from Coprinopsis cinerea drives lytic polysaccharide monooxygenase action. Appl. Environ. Microbiol 84, e00156-00118 (2018)

25. Garajova, S. et al. Single-domain flavoenzymes trigger lytic polysaccharide monooxygenases for oxidative degradation of cellulose. Sci. Rep. 6, 28276 (2016).

26. Phillips, C. M., Beeson, W. T., Cate, J. H. \& Marletta, M. A. Cellobiose dehydrogenase and a copper-dependent polysaccharide monooxygenase potentiate cellulose degradation by Neurospora crassa. ACS Chem. Biol. 6 , 1399-1406 (2011).

27. Kracher, D. et al. Extracellular electron transfer systems fuel cellulose oxidative degradation. Science 352, 1098-1101 (2016)

28. Miyauchi, S. et al. Conserved white-rot enzymatic mechanism for wood decay in the Basidiomycota genus Pycnoporus. DNA Res. 27, dsaa011 (2020).

29. Kracher, D. et al. Polysaccharide oxidation by lytic polysaccharide monooxygenase is enhanced by engineered cellobiose dehydrogenase. FEBS J. 287, 897-908 (2020).

30. Berrin, J. G., Rosso, M. N. \& Abou Hachem, M. Fungal secretomics to probe the biological functions of lytic polysaccharide monooxygenases. Carbohydr. Res. 448, 155-160 (2017).

31. Nekiunaite, L., Arntzen, M. Ø., Svensson, B., Vaaje-Kolstad, G. \& Abou Hachem, M. Lytic polysaccharide monooxygenases and other oxidative enzymes are abundantly secreted by Aspergillus nidulans grown on different starches. Biotechnol. Biofuels 9, 187 (2016)

32. Winkler, A. et al. Structural and mechanistic studies reveal the functional role of bicovalent flavinylation in berberine bridge enzyme. J. Biol. Chem. 284, 19993-20001 (2009)

33. Daniel, B. et al. Oxidation of monolignols by members of the berberine bridge enzyme family suggests a role in plant cell wall metabolism. J. Biol. Chem. 290, 18770-18781 (2015).

34. Bennati-Granier, C. et al. Substrate specificity and regioselectivity of fungal AA9 lytic polysaccharide monooxygenases secreted by Podospora anserina. Biotechnol. Biofuels 8, 90 (2015).

35. Frandsen, K. E. H. et al. The molecular basis of polysaccharide cleavage by lytic polysaccharide monooxygenases. Nat. Chem. Biol. 12, 298-303 (2016).

36. Frandsen, K. E. H. et al. Identification of the molecular determinants driving the substrate specificity of fungal lytic polysaccharide monooxygenases (LPMOs). J. Biol. Chem. 296, 100086 (2021).

37. Kracher, D., Andlar, M., Furtmuller, P. G. \& Ludwig, R. Active-site copper reduction promotes substrate binding of fungal lytic polysaccharide monooxygenase and reduces stability. J. Biol. Chem. 293, 1676-1687 (2018).

38. Bissaro, B., Kommedal, E., Røhr, Å. K. \& Eijsink, V. G. H. Controlled depolymerization of cellulose by light-driven lytic polysaccharide oxygenases. Nat. Commun. 11, 890 (2020).

39. Winkler, A., Kutchan, T. M. \& Macheroux, P. 6-S-cysteinylation of bicovalently attached FAD in berberine bridge enzyme tunes the redox potential for optimal activity. J. Biol. Chem. 282, 24437-24443 (2007).

40. Huang, C.-H. et al. Functional roles of the 6-S-cysteinyl, 8alpha-N1-histidyl FAD in glucooligosaccharide oxidase from Acremonium strictum. J. Biol. Chem. 283, 30990-30996 (2008).

41. Dermoun, Z. et al. The NADP-reducing hydrogenase from Desulfovibrio fructosovorans: functional interaction between the C-terminal region of HndA and the N-terminal region of HndD subunits. Biochim Biophys. Acta 1556, 217-225 (2002).

42. Kutchan, T. M. \& Dittrich, H. Characterization and mechanism of the berberine bridge enzyme, a covalently flavinylated oxidase of benzophenanthridine alkaloid biosynthesis in plants. J. Biol. Chem. 270 24475-24481 (1995).

43. Romero, E., Gomez Castellanos, J. R., Gadda, G., Fraaije, M. W. \& Mattevi, A. Same substrate, many reactions: oxygen activation in flavoenzymes. Chem. Rev. 118, 1742-1769 (2018).

44. Zafred, D. et al. Rationally engineered flavin-dependent oxidase reveals steric control of dioxygen reduction. FEBS J. 282, 3060-3074 (2015).
45. Hernández-Ortega, A. et al. Role of active site histidines in the two halfreactions of the aryl-alcohol oxidase catalytic cycle. Biochemistry 51, 6595-6608 (2012)

46. Johansen, K. S. Lytic polysaccharide monooxygenases: the microbial power tool for lignocellulose degradation. Trends Plant Sci. 21, 926-936 (2016)

47. Loose, J. S. M. et al. Multipoint precision binding of substrate protects lytic polysaccharide monooxygenases from self-destructive off-pathway processes. Biochemistry 57, 4114-4124 (2018).

48. Massey, V. Activation of molecular oxygen by flavins and flavoproteins. J. Biol Chem. 269, 22459-22462 (1994)

49. Tan, T.-C. et al. Structural basis for cellobiose dehydrogenase action during oxidative cellulose degradation. Nat. Commun. 6, 7542 (2015).

50. Breslmayr, E. et al. Protein conformational change is essential for reductive activation of lytic polysaccharide monooxygenase by cellobiose dehydrogenase. ACS Catal. 10, 4842-4853 (2020).

51. Katoh, K., Rozewicki, J. \& Yamada, K. D. MAFFT online service: multiple sequence alignment, interactive sequence choice and visualization. Brief. Bioinform. 20, 1160-1166 (2019).

52. Castresana, J. Selection of conserved blocks from multiple alignments for their use in phylogenetic analysis. Mol. Biol. Evol. 17, 540-552 (2000).

53. Lemoine, F. et al. NGPhylogeny.fr: new generation phylogenetic services for non-specialists. Nucleic Acids Res. 47, W260-W265 (2019).

54. Letunic, I. \& Bork, P. Interactive Tree Of Life (iTOL) v4: recent updates and new developments. Nucleic Acids Res. 47, W256-W259 (2019).

55. Crooks, G. E., Hon, G., Chandonia, J. M. \& Brenner, S. E. WebLogo: a sequence logo generator. Genome Res. 14, 1188-1190 (2004).

56. Bunkoczi, G. et al. MRage: automated molecular replacement. Acta Crystallogr. Sect. D 69, 2276-2286 (2013).

57. Terwilliger, T. C. et al. Iterative model building, structure refinement and density modification with the PHENIX AutoBuild wizard. Acta Crystallogr. Sect. D 64, 61-69 (2008).

58. Adams, P. D. et al. PHENIX: a comprehensive Python-based system for macromolecular structure solution. Acta Crystallogr. Sect. D. Biol. Crystallogr. 66, 213-221 (2010)

59. Emsley, P., Lohkamp, B., Scott, W. G. \& Cowtan, K. Features and development of Coot. Acta Crystallogr. Sect. D. Biol. Crystallogr. 66, 486-501 (2010).

60. Williams, C. J. et al. MolProbity: more and better reference data for improved all-atom structure validation. Protein Sci. 27, 293-315 (2018).

61. Wood, T. M. In Methods in Enzymology (ed^(eds)). (Academic Press, 1988)

62. Westereng, B. et al. Efficient separation of oxidized cello-oligosaccharides generated by cellulose degrading lytic polysaccharide monooxygenases. J. Chromatogr. A 1271, 144-152 (2013).

\section{Acknowledgements}

This study was supported by a Novo Nordisk Foundation Post-doc grant within the Biotechnology Based Synthesis and Production Research Programme to MHM (NNF17OC0025642). F.F. and D.H.W. were funded by The Novo Nordisk Foundation Grant No. NNF10CC1016517. NMR spectra were acquired at the NMR center DTU supported by the Villum and Carlsberg Foundations. J.G.B., B.G. (Bruno G) and F.B. (Frédéric B) thank IM2B (Institut de Microbiologie, Bioénergies et Biotechnologie). The DSC instrument is funded by the Carlsberg Foundation Grant (2013-01-0112) to M.A.H The authors are also grateful to the EPR facilities available at the French EPR network (IR CNRS 3443) and the Aix-Marseille University EPR center. Prof. Peter Westh and Dr. Jeppi Kari are acknowledged for discussion on the redox-activities of AA7.

\section{Author contributions}

M.H.M., J.G.B. and M.A.H. conceived the research and designed the experiments. M.H.M. and F.F. crystallised the enzymes, F.F. collected the crystallographic data and generated the first models, M.H.M. refined the structures with F.F. M.H.M. performed the sequence and phylogenetic analysis with help from V.L. Recombinant enzyme production was performed by M.H.M. and M.H. M.H.M. generated the initial biochemical characterisation except for PbChi7A. M.H.M. and T.S.N. carried out the kinetic analyses and TSN measured the $\mathrm{pH}$, temperature and DCIP activity profiles. All biochemical data on $\mathrm{PbChi7A}$ were generated by O.R. and T.V.V. ERM provided the funding and the facilities for the work on $\mathrm{PbChi7A}$. The NMR analyses were performed and interpreted by S.M. MHM performed the cellulose degradation assays and the HPAEC-PAD with support from S.G. M.H.M. and B.B. designed the SOD/HRP effect experiments, which were carried out by M.H.M. B.B. and M.H.M., F.B. and B.G. performed the EPR analysis D.H.W. and B.H. provided funding and interpretation of the crystallography and sequence analysis part. M.A.H. and M.H.M. analysed the data and wrote the first version of the manuscript. J.G.B. and B.B. contributed to the writing of later versions of the manuscript. All authors contributed and approved the final version of the manuscript.

\section{Competing interests}

A provisional patent has been filed based on data from this paper by M.H.M., J.G.B. and M.A.H. The other authors declare that they have no competing interests. 


\section{Additional information}

Supplementary information The online version contains supplementary material available at https://doi.org/10.1038/s41467-021-22372-0.

Correspondence and requests for materials should be addressed to J.-G.B. or M.A.H.

Peer review information Nature Communications thanks Igor Polikarpov and the other, anonymous, reviewer(s) for their contribution to the peer review of this work. Peer reviewer reports are available.

Reprints and permission information is available at http://www.nature.com/reprints

Publisher's note Springer Nature remains neutral with regard to jurisdictional claims in published maps and institutional affiliations. (c) (i) Open Access This article is licensed under a Creative Commons Attribution 4.0 International License, which permits use, sharing, adaptation, distribution and reproduction in any medium or format, as long as you give appropriate credit to the original author(s) and the source, provide a link to the Creative Commons license, and indicate if changes were made. The images or other third party material in this article are included in the article's Creative Commons license, unless indicated otherwise in a credit line to the material. If material is not included in the article's Creative Commons license and your intended use is not permitted by statutory regulation or exceeds the permitted use, you will need to obtain permission directly from the copyright holder. To view a copy of this license, visit http://creativecommons.org/ licenses/by/4.0/.

(C) The Author(s) 2021 